Bulletin of the Section of Logic

Volume 49/3 (2020), pp. 291-324

http://dx.doi.org/10.18778/0138-0680.2020.14

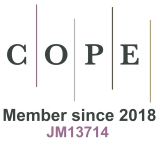

Shokoofeh Ghorbani

\title{
EQUALITY LOGIC
}

\begin{abstract}
In this paper, we introduce and study a corresponding logic to equality-algebras and obtain some basic properties of this logic. We prove the soundness and completeness of this logic based on equality-algebras and local deduction theorem. We show that this logic is regularly algebraizable with respect to the variety of equality-algebras but it is not Fregean. Then we introduce the concept of (prelinear) equality $\triangle$-algebras and investigate some related properties. Also, we study $\triangle$-deductive systems of equality $\triangle$-algebras. In particular, we prove that every prelinear equality $\triangle$-algebra is a subdirect product of linearly ordered equality $\triangle$ algebras. Finally, we construct prelinear equality $\triangle$ logic and prove the soundness and strong completeness of this logic respect to prelinear equality $\triangle$-algebras.
\end{abstract}

Keywords: Many-valued logic, equality logic, completness, prelinear equality $\triangle^{-}$ algebra, prelinear equality $\triangle$ logic.

\section{Introduction}

Novák introduced the concept of EQ-algebras in [17] as candidates for a possible algebraic semantics of fuzzy-type theory (see [16]). These algebras are meet semilattices endowed with two additional binary operations: fuzzy equality and multiplication. Implication is derived from the fuzzy equality and it is not a residuation with respect to multiplication. Consequently, EQ-algebras is a generalization of residuated lattices in the sense that each residuated lattice is an EQ-algebra but not vice-versa.

Presented by: Janusz Czelakowski

Received: December 24, 2019

Published online: August 15, 2020

(C) Copyright for this edition by Uniwersytet Łódzki, Łódź 2020 
Dyba and Novák introduced EQ-logic in [9] as a specific formal logic in which the basic connective is fuzzy equality and the implication is derived from the fuzzy equality. They formulated the basic EQ-logic and proved the completeness of this logic. Also, see $[19,10,11]$.

Recently, Dyba and et all in [8], introduced and studied the prelinear $\mathrm{EQ}_{\triangle}$-algebras and the corresponding propositional EQ $\triangle$-logic.

As Jenei mentioned in [13], if the product operation in EQ-algebras is replaced by another binary operation smaller or equal than the original (viewed as a two-place function) we still obtain an EQ-algebra. This fact might make it difficult to obtain certain algebraic results. For this reason, Jenei introduced a new structure in [13], called equality-algebra, to find something similar to EQ-algebras but without a product. The equality-algebras have two binary operations meet and equivalence, and a constant 1. Jenei proved the term equivalence of the closed algebras to BCK-meet-semilattices. In [23], F. Zebardast and et all studied and proved that there are relations among equality algebras and some of other logical algebras such as residuated lattice, MTL-algebra, BL-algebra, MValgebra, Hertz-algebra, Heyting-algebra, Boolean-algebra, EQ-algebra and hoop-algebra. Some types of filters of equality algebras are introduced in [3]. Since then many researchers have worked on this area (see $[4,6,14,12])$.

In this paper, we will show that equality-algebras are semantics of fuzzytype theory. In the next section, we review some notions which are needed in the sequel. In section 3, the corresponding equality logic is constructed and some related properties are proved. Also, the soundness and completeness of this logic are proved. We prove that this logic is regularly algebraizable with respect to the variety of the equality-algebras. In section 4 , we investigate (prelinear) equality $\triangle$-algebras and $\triangle$-deductive systems on equality $\triangle$-algebras. We obtain some related results. Finally in section 5, we introduce prelinear equality $\triangle$ logic and prove strong completeness.

\section{Preliminaries}

In this section, we recall the basic definitions and some known results about equality-algebras that we need in the rest of the paper.

Definition 2.1 ([13]). An equality-algebra is an algebra $\mathcal{A}=(A, \wedge, \sim, 1)$ of the type $(2,2,0)$ such that satisfies the following axioms for all $x, y, z \in$ $A$ : 
(E1) $(A, \wedge, 1)$ is a meet-semilattice with top element 1 ,

(E2) $x \sim y=y \sim x$,

(E3) $x \sim x=1$,

(E4) $x \sim 1=x$,

(E5) $x \leq y \leq z$ implies $x \sim z \leq y \sim z$ and $x \sim z \leq x \sim y$,

(E6) $x \sim y \leq(x \wedge z) \sim(y \wedge z)$,

(E7) $x \sim y \leq(x \sim z) \sim(y \sim z)$.

The operation $\wedge$ is called meet (infimum) and $\sim$ is an equality operation. We write $x \leq y$ (and $y \geq x$ ) iff $x \wedge y=x$. Define the following two derived operations, the implication and the equivalence operation of the equalityalgebra $\mathcal{A}$ by

(I) $x \rightarrow y=x \sim(x \wedge y)$,

(II) $x \leftrightarrow y=(x \rightarrow y) \wedge(y \rightarrow x)$.

An equality-algebra $\mathcal{A}=(A, \wedge, \sim, 1)$ is bounded if there exists an element $0 \in A$ such that $0 \leq x$, for all $x \in A$.

Proposition 2.2 ([13]). Let $\mathcal{A}=(A, \wedge, \sim, 1)$ be an equality-algebra and consider

(E5a) $x \sim(x \wedge y \wedge z) \leq x \sim(x \wedge y)$,

(E5a') $x \rightarrow(y \wedge z) \leq x \rightarrow y$,

Then (E5) is equivalent to (E5a), which in turn is equivalent to (E5a').

Definition $2.3([23])$. Let $\mathcal{A}=(A, \wedge, \sim, 1)$ be an equality-algebra.

(1) Then $\mathcal{A}$ is called prelinear, if 1 is the unique upper bound of the set $\{x \rightarrow y, y \rightarrow x\}$ for all $x, y \in A$.

(2) A lattice equality-algebra is an equality-algebra which is a lattice.

Theorem 2.4 ([8]). Any prelinear equality-algebra is a distributive lattice. 
Proposition 2.5 ([13, 23]). Let $\mathcal{A}=(A, \wedge, \sim, 1)$ be an equality-algebra. Then the following hold for all $x, y, z \in A$ :

(1) $x \sim y \leq x \leftrightarrow y \leq x \rightarrow y$,

(2) $x \leq(x \sim y) \sim y$,

(3) $x \sim y=1$ iff $x=y$,

(4) $x \rightarrow y=1$ iff $x \leq y$,

(5) $x \rightarrow y=1$ and $y \rightarrow x=1$ implies $x=y$,

(6) $1 \rightarrow x=x, x \rightarrow 1=1$ and $x \rightarrow x=1$,

(7) $x \leq y \rightarrow x$,

(8) $x \leq(x \rightarrow y) \rightarrow y$,

(9) $x \rightarrow y \leq(y \rightarrow z) \rightarrow(x \rightarrow z)$,

(10) $x \leq y \rightarrow z$ iff $y \leq x \rightarrow z$,

(11) $x \rightarrow(y \rightarrow z)=y \rightarrow(x \rightarrow z)$,

(12) $x \leftrightarrow x=1,1 \leftrightarrow x=x$,

(13) $x \leq y$ implies $x \leftrightarrow y=y \rightarrow x=y \sim x$,

(14) $x \leq y$ implies $x \leq x \sim y$,

(15) $x \leq y$ implies that $z \rightarrow x \leq z \rightarrow y$ and $y \rightarrow z \leq x \rightarrow z$,

(16) if $\mathcal{A}$ is a lattice equality-algebra, then $(x \vee y) \rightarrow z=(x \rightarrow z) \wedge(y \rightarrow z)$,

(17) if $\mathcal{A}$ is a prelinear equality-algebra, then $x \sim y=(x \rightarrow y) \wedge(y \rightarrow x)$.

Definition 2.6 ([13]). Let $\mathcal{A}=(A, \wedge, \sim, 1)$ be an equality-algebra and $F$ be a subset of $A$. Then $F$ is called a deductive system of $\mathcal{A}$ if for all $x, y \in A$,

(i) $1 \in F$,

(ii) if $x \in F$ and $x \leq y$, then $y \in F$,

(iii) if $x \in F$ and $x \sim y \in F$, then $y \in F$. 
Proposition $2.7([13])$. Let $\mathcal{A}=(A, \wedge, \sim, 1)$ be an equality-algebra and $F$ be a subset of $A$. Then $F$ is a deductive system of $\mathcal{A}$ if and only if

(i) $1 \in F$,

(ii) if $x \in F$ and $x \rightarrow y \in F$, then $y \in F$.

A deductive system $F$ of an equality-algebra $\mathcal{A}=(A, \wedge, \sim, 1)$ is called a proper deductive system if $F \neq A$. If $\mathcal{A}$ is a bounded equality-algebra, then a deductive system is proper if and only if it does not contain 0 (see [3]).

DEFinition 2.8 ([13]). An equivalence relation $\theta$ on an equality-algebra $\mathcal{A}=(A, \wedge, \sim, 1)$ is called congruence, if $(x, z),(y, w) \in \theta$, then $(x \sim y$, $z \sim w),(x \wedge y, z \wedge w) \in \theta$.

Proposition $2.9([6,13])$. Let $F$ be a deductive system of an equalityalgebra $\mathcal{A}=(A, \wedge, \sim, 1)$. Define the relation $\theta_{\vec{F}}$ and $\theta_{F}$ as follows:

$$
(x, y) \in \theta_{\vec{F}} \text { iff }\{x \rightarrow y, y \rightarrow x\} \subseteq F
$$

and

$$
(x, y) \in \theta_{F} \text { iff } x \sim y \in F,
$$

then $\theta_{\vec{F}}$ and $\theta_{F}$ are congruence and $\theta_{\vec{F}}=\theta_{F}$.

Proposition $2.10([6,13])$. Let $F$ be a deductive system of an equalityalgebra $\mathcal{A}=(A, \wedge, \sim, 1)$ and $A / \theta_{F}=\{[x]: x \in A\}$, where $[x]=\{y \in A$ : $\left.(x, y) \in \theta_{F}\right\}$. Then $\mathcal{A} / \theta_{\mathcal{F}}=\left(A / \theta_{F}, \wedge, \sim, 1\right)$ is an equality-algebra, where for every $x, y \in A, 1:=[1],[x] \wedge[y]:=[x \wedge y]$ and $[x] \sim[y]:=[x \sim y]$.

Definition $2.11([3])$. A proper deductive system $F$ of an equality-algebra $\mathcal{A}=(A, \wedge, \sim, 1)$ is called a prime deductive system if $x \rightarrow y \in F$ or $y \rightarrow x \in F$ for all $x, y \in A$.

TheOREM 2.12 ([3]). Let $F$ be a proper deductive system of prelinear equality-algebra $\mathcal{A}=(A, \wedge, \sim, 1)$. Then the following statements are equivalent:

(i) $F$ is a prime deductive system,

(ii) for each $x, y \in A$, if $x \vee y \in F$, then $x \in F$ or $y \in F$,

(iii) $\mathcal{A} / \theta_{F}$ is a chain. 
Definition $2.13([21])$. Let $\mathcal{A}=(A, \wedge, \sim, 1)$ be an equality-algebra. The mapping $\tau: A \rightarrow A$ is called a very true operator, if it satisfies the following conditions:

(V1) $\tau(1)=1$,

(V2) $\tau(x) \leq x$,

(V3) $\tau(x) \leq \tau(\tau(x))$,

(V4) $\tau(x \sim y) \leq \tau(x) \sim \tau(y)$,

$(V 5) \tau(x \wedge y)=\tau(x) \wedge \tau(y)$.

\section{Equality logic}

In this section, we introduce and study a propositional equality logic and we obtain some its properties.

DeFinition 3.1.

(i) The language of propositional equality logic $\mathcal{E} \mathcal{L}$ has propositional variables $p, q, r, \ldots$ binary connectives $\Pi$, $\equiv$ and a truth (logical) constant $T$.

(ii) Formulas of $\mathcal{E} \mathcal{L}$ are defined in the following way: each propositional variable is a formula, $\top$ is a formula and if $\varphi, \psi$ are formulas, then $\varphi \sqcap$ $\psi$ (conjunction) and $\varphi \equiv \psi$ are formulas. Implication and equivalence connectives are defined as the following short:

$$
\varphi \Rightarrow \psi:=(\varphi \sqcap \psi) \equiv \varphi, \quad \varphi \Leftrightarrow \psi:=(\varphi \Rightarrow \psi) \sqcap(\psi \Rightarrow \varphi) .
$$

The set of all formulas of $\mathcal{E} \mathcal{L}$ is denoted by $\mathcal{F}$.

(iii) The following formulas are axioms of $\mathcal{E} \mathcal{L}$ :

(EL1) $\varphi \sqcap \varphi \equiv \varphi$,

(EL2) $\varphi \sqcap \psi \equiv \psi \sqcap \varphi$,

(EL3) $(\varphi \sqcap \psi) \sqcap \chi \equiv \varphi \sqcap(\psi \sqcap \chi)$,

(EL4) $\varphi \sqcap \top \equiv \varphi$,

(EL5) $(\varphi \equiv \top) \equiv \varphi$,

$(\operatorname{EL} 6)(\varphi \equiv \psi) \equiv(\psi \equiv \varphi)$, 
(EL7) $((\varphi \sqcap \psi \sqcap \chi) \equiv \varphi) \Rightarrow((\varphi \sqcap \psi) \equiv \varphi)$,

(EL8) $(\varphi \equiv \psi) \Rightarrow(\varphi \sqcap \chi \equiv \psi \sqcap \chi)$,

(EL9) $(\varphi \equiv \psi) \Rightarrow((\varphi \equiv \chi) \equiv(\psi \equiv \chi))$.

(iv) The inference rules are :

(EA) from $\psi$ and $\varphi \equiv \psi$, we infer $\varphi$,

(MP) from $\varphi$ and $\varphi \Rightarrow \psi$, we infer $\psi$.

The rule (EA) is the equanimity rule (cf. [18]) and (MP) is the modus ponens rule for formulas.

Definition 3.2. Let $\mathcal{A}=(A, \wedge, \sim, 1)$ be an equality-algebra. An $\mathcal{A}$ evaluation of formulas is a mapping $e: \mathcal{F} \rightarrow A$, defined as follows:

(1) $e(\top)=1$,

(2) $e(\varphi \sqcap \psi)=e(\varphi) \wedge e(\psi)$,

(3) $e(\varphi \equiv \psi)=e(\varphi) \sim e(\psi)$,

for all formulas $\varphi, \psi \in \mathcal{F}$. A formula $\varphi$ is a $\mathcal{A}$-tautology if $e(\varphi)=1$ for each $\mathcal{A}$ - evaluation $e: \mathcal{F} \rightarrow A$.

Lemma 3.3. All axioms of $\mathcal{E} \mathcal{L}$ are $\mathcal{A}$-tautologies for all equality-algebra $\mathcal{A}=(A, \wedge, \sim, 1)$.

Proof: Suppose that $\mathcal{A}=(A, \wedge, \sim, 1)$ is an arbitrary equality-algebra and $e: \mathcal{F} \rightarrow A$ is an arbitrary $\mathcal{A}$-evaluation.

(EL1) By (E1) and (E2), we have $e(\varphi \sqcap \varphi \equiv \varphi)=e(\varphi \sqcap \varphi) \sim e(\varphi)=$ $(e(\varphi) \wedge e(\varphi)) \sim e(\varphi)=e(\varphi) \sim e(\varphi)=1$.

(EL2) Using (E1) and (E3), $e(\varphi \sqcap \top \equiv \varphi)=e(\varphi \sqcap \top) \sim e(\varphi)=(e(\varphi) \wedge$ $e(\top)) \sim e(\varphi)=(e(\varphi) \wedge 1) \sim e(\varphi)=e(\varphi) \sim e(\varphi)=1$ by $(\mathrm{A} 2)$.

Similarly, we can prove the (EL3)-(EL9).

Lemma 3.4. The inference rules of propositional equality logic $\mathcal{E} \mathcal{L}$ sound in the following sense: Let $e: \mathcal{F} \rightarrow A$ be an $\mathcal{A}$-evaluation where $\mathcal{A}$ is an equality-algebra:

(1) if $\varphi$ and $\varphi \equiv \psi$ are $\mathcal{A}$-tautology, then $\psi$ is also $\mathcal{A}$-tautology,

(2) if $\varphi$ and $\varphi \Rightarrow \psi$ are $\mathcal{A}$-tautology, then $\psi$ is also $\mathcal{A}$-tautology. 
Proof:

(1) Suppose that $e(\varphi)=1$ and $e(\varphi \equiv \psi)=1$. Then $1=e(\psi) \sim 1=e(\psi)$ by $(\mathrm{E} 4)$.

Similarly, we can prove (2).

TheOREM 3.5 (Soundness). The propositional equality logic $\mathcal{E} \mathcal{L}$ is sound.

Proof: It follows from Lemma 3.3 and Lemma 3.4.

A proof in propositional equality logic $\mathcal{E} \mathcal{L}$ is a sequence $\varphi_{1}, \ldots, \varphi_{n}$ of formulas such that each $\varphi_{i}$ either is an axiom of $\mathcal{E} \mathcal{L}$ or follows from some preceding $\varphi_{j}, \varphi_{k}(j, k<i)$ by inference rules. A formula is provable (notation $\vdash \varphi$ ) if it is the least member a proof in propositional equality logic $\mathcal{E} \mathcal{L}$. By Theorem 3.5, each provable formula in propositional equality logic $\mathcal{E} \mathcal{L}$ is $A$-tautology for all equality-algebra $\mathcal{A}$.

A theory over propositional equality $\operatorname{logic} \mathcal{E} \mathcal{L}$ is any subset $\Gamma \subseteq \mathcal{F}$. A proof in a theory $\Gamma$ is a sequence $\varphi_{1}, \ldots, \varphi_{n}$ of formulas whose each member is either an axiom $\mathcal{E} \mathcal{L}$ or a member of $\Gamma$ (spacial axiom) or follows from some preceding members of the sequence using the inference rules. $\Gamma \vdash \varphi$ means that $\varphi$ is provable in $\Gamma$, that is the last member of a proof in $\Gamma$. An $\mathcal{A}$-evaluation $e$ is a model of $\Gamma$, if $e(\varphi)=1$ for each $\varphi \in \Gamma$. If $\Gamma=\{\varphi\}$, then we write $\varphi \vdash \psi$ instead of $\{\varphi\} \vdash \psi$.

In the following, we will verify provability of several formulas in propositional equality logic $\mathcal{E} \mathcal{L}$.

Proposition 3.6. Let $\varphi, \psi, \chi \in \mathcal{F}$ be formulas. $\mathcal{E} \mathcal{L}$ proves the following properties of equality:
(1) $\vdash \varphi \equiv \varphi$,
(2) $\varphi \vdash \varphi \equiv \top$,
(3) $\varphi \equiv \top \vdash \varphi$,
(4) $\varphi \equiv \psi \vdash \psi \equiv \varphi$,
(5) $\varphi \sqcap \psi \equiv \chi \vdash \psi \sqcap \varphi \equiv \chi$,
(6) $\varphi \equiv \psi \vdash(\varphi \equiv \chi) \equiv(\psi \equiv \chi)$,
(7) $\{\varphi \equiv \psi, \psi \equiv \chi\} \vdash \varphi \equiv \chi$, 
(8) $\varphi \equiv \psi \vdash(\chi \equiv \varphi) \equiv(\chi \equiv \psi)$,

(9) $\varphi \equiv \psi \vdash(\chi \sqcap \varphi) \equiv(\chi \sqcap \psi)$,

(10) $\{\psi, \psi \equiv \varphi\} \vdash \varphi$.

\section{PROOF:}

(1) We have $\vdash(\varphi \sqcap \varphi \equiv \varphi) \Rightarrow((\varphi \sqcap \varphi \equiv \varphi) \equiv(\varphi \equiv \varphi))$ by (EL9). Applying (EL1) and (MP), we get $\vdash(\varphi \sqcap \varphi \equiv \varphi) \equiv(\varphi \equiv \varphi)$. Using (EL1) and (EA), we obtain $\vdash \varphi \equiv \varphi$.

(2) By (EL6), we have $\vdash((\varphi \equiv \top) \equiv \varphi) \equiv(\varphi \equiv(\varphi \equiv \top))$. Applying (EL5) and then (EA), we obtain $\vdash \varphi \equiv(\varphi \equiv T)$. Using assumption and (EA), we get the result.

(3) It follows from (EL5), assumption and (EA).

(4) By assumption, (EL6) and (EA), we obtain the result.

(5) Using (EL9), we have $\vdash(\varphi \sqcap \psi \equiv \psi \sqcap \varphi) \Rightarrow((\varphi \sqcap \psi \equiv \chi) \equiv(\psi \sqcap \varphi \equiv \chi))$. Applying (EL2) and (MP), we get $\vdash(\varphi \sqcap \psi \equiv \chi) \equiv(\psi \sqcap \varphi \equiv \chi)$. Using assumption and (EA), we have $\varphi \sqcap \psi \equiv \chi \vdash \psi \sqcap \varphi \equiv \chi$.

(6) It is immediate consequence of (EL9).

(7) We have $\varphi \equiv \psi \vdash(\varphi \equiv \chi) \equiv(\psi \equiv \chi)$ by assumption and part (6). Using part (4), we get $\varphi \equiv \psi \vdash(\psi \equiv \chi) \equiv(\varphi \equiv \chi)$. By assumption and (EA), we get the result.

(8) By assumption, part (4) and then part (6), we have $\varphi \equiv \psi \vdash(\psi \equiv$ $\chi) \equiv(\varphi \equiv \chi)$. Since we have $\vdash((\psi \equiv \chi) \equiv(\varphi \equiv \chi)) \Rightarrow(((\psi \equiv \chi) \equiv$ $(\chi \equiv \psi)) \equiv((\varphi \equiv \chi) \equiv(\chi \equiv \psi)))$ by $($ EL9 $)$, then $\vdash((\psi \equiv \chi) \equiv$ $(\chi \equiv \psi)) \equiv((\varphi \equiv \chi) \equiv(\chi \equiv \psi))$ by (MP). Using (EL6), we have $\vdash(\psi \equiv \chi) \equiv(\chi \equiv \psi)$. So by $(\mathrm{EA})$, we get $\vdash(\varphi \equiv \chi) \equiv(\chi \equiv \psi)$. Applying $($ EL9 $) \vdash((\varphi \equiv \chi) \equiv(\chi \equiv \varphi)) \Rightarrow(((\varphi \equiv \chi) \equiv(\chi \equiv \psi)) \equiv$ $((\chi \equiv \varphi) \equiv(\chi \equiv \psi)))$. By $(\mathrm{EL} 6) \vdash(\varphi \equiv \chi) \equiv(\chi \equiv \varphi)$ and (MP), we get $\vdash((\varphi \equiv \chi) \equiv(\chi \equiv \psi)) \equiv((\chi \equiv \varphi) \equiv(\chi \equiv \psi))$. Hence by (EA), we obtain $\varphi \equiv \psi \vdash(\chi \equiv \varphi) \equiv(\chi \equiv \psi)$.

(9) It follows from (EL8), (EL2) and part (8).

(10) Using assumptions, part (4) and (EA), we get result. 
Proposition 3.7. Let $\varphi, \varphi_{1}, \psi, \psi_{1}, \chi, \chi_{1} \in \mathcal{F}$ be formulas. $\mathcal{E} \mathcal{L}$ proves the following:

(1) $\left\{\varphi \sqcap \psi \equiv \chi, \varphi \equiv \varphi_{1}\right\} \vdash \varphi_{1} \sqcap \psi \equiv \chi$,

(2) $\left\{(\varphi \equiv \psi) \equiv \chi, \varphi \equiv \varphi_{1}\right\} \vdash\left(\varphi_{1} \equiv \psi\right) \equiv \chi$,

(3) $\left\{\varphi \Rightarrow(\psi \equiv \chi), \psi \equiv \psi_{1}\right\} \vdash \varphi \Rightarrow\left(\psi_{1} \equiv \chi\right)$,

(4) $\left\{\varphi \Rightarrow(\psi \equiv \chi), \chi \equiv \chi_{1}\right\} \vdash \varphi \Rightarrow\left(\psi \equiv \chi_{1}\right)$,

(5) $\left\{\varphi \Rightarrow \psi, \varphi \equiv \varphi_{1}\right\} \vdash \varphi_{1} \Rightarrow \psi$,

(6) $\{\varphi \Rightarrow(\psi \equiv \chi), \psi\} \vdash \varphi \Rightarrow \chi$,

(7) $\{\varphi, \psi\} \vdash \varphi \sqcap \psi$,

(8) $\varphi \equiv \psi \vdash \varphi \Rightarrow \psi$,

(9) $\left\{(\varphi \equiv \psi) \Rightarrow \chi, \varphi \equiv \varphi_{1}\right\} \vdash\left(\varphi_{1} \equiv \psi\right) \Rightarrow \chi$.

\section{PROOF:}

(1) Suppose that $\Gamma=\left\{\varphi \sqcap \psi, \varphi \equiv \varphi_{1}\right\}$. By assumption $\Gamma \vdash \varphi \equiv \varphi_{1}$, (EL8) and (MP), we obtain $\Gamma \vdash(\varphi \sqcap \psi) \equiv\left(\varphi_{1} \sqcap \psi\right)$. Using (EL9), $\vdash\left((\varphi \sqcap \psi) \equiv\left(\varphi_{1} \sqcap \psi\right)\right) \Rightarrow\left((\varphi \sqcap \psi \equiv \chi) \equiv\left(\varphi_{1} \sqcap \psi \equiv \chi\right)\right)$ and (MP), we have $\Gamma \vdash(\varphi \sqcap \psi \equiv \chi) \equiv\left(\varphi_{1} \sqcap \psi \equiv \chi\right)$. Applying assumption $\Gamma \vdash \varphi \sqcap \psi \equiv \chi$ and (EA), we get the result.

(2) Let $\Gamma=\left\{(\varphi \equiv \psi) \equiv \chi, \varphi \equiv \varphi_{1}\right\}$. Using assumption $\Gamma \vdash \varphi \equiv$ $\varphi_{1},\left(\right.$ EL9) and (MP), we have $\Gamma \vdash(\varphi \equiv \psi) \equiv\left(\varphi_{1} \equiv \psi\right)$. Since $\left.\vdash\left((\varphi \equiv \psi) \equiv\left(\varphi_{1} \equiv \psi\right)\right) \equiv((\varphi \equiv \psi) \equiv \chi) \equiv\left(\left(\varphi_{1} \equiv \psi\right) \equiv \chi\right)\right)$ by (EL9), then $\Gamma \vdash(\varphi \equiv \psi) \equiv \chi) \equiv\left(\left(\varphi_{1} \equiv \psi\right) \equiv \chi\right)$. Applying assumption $\Gamma \vdash(\varphi \equiv \psi) \equiv \chi$ and $(\mathrm{EA})$, we have $\Gamma \vdash\left(\left(\varphi_{1} \equiv \psi\right) \equiv \chi\right)$.

(3) Suppose that $\Gamma=\left\{\varphi \Rightarrow(\psi \equiv \chi), \psi \equiv \psi_{1}\right\}$. Since $\Gamma \vdash \psi \equiv \psi_{1}$, then $\Gamma \vdash(\psi \equiv \chi) \equiv\left(\psi_{1} \equiv \chi\right)$ by Proposition 3.6 part (6). We have $\Gamma \vdash(\varphi \sqcap(\psi \equiv \chi)) \equiv \varphi$ by assumption. Hence $\Gamma \vdash\left(\varphi \sqcap\left(\psi_{1} \equiv \chi\right)\right) \equiv \varphi$ by part (1), that is $\Gamma \vdash \varphi \Rightarrow\left(\psi_{1} \equiv \chi\right)$.

(4) Let $\Gamma=\left\{\varphi \Rightarrow(\psi \equiv \chi), \chi \equiv \chi_{1}\right\}$. By assumption $\Gamma \vdash \chi \equiv \chi_{1}$ and Proposition 3.6 part $(8)$ we get $\Gamma \vdash(\psi \equiv \chi) \equiv\left(\psi \equiv \chi_{1}\right)$. 
Applying part (1) and assumption $\Gamma \vdash(\varphi \sqcap(\psi \equiv \chi)) \equiv \varphi$, we obtain $\Gamma \vdash\left(\varphi \sqcap\left(\psi \equiv \chi_{1}\right)\right) \equiv \varphi$. Hence $\Gamma \vdash \varphi \Rightarrow\left(\psi \equiv \chi_{1}\right)$.

(5) It follows from assumptions and part (1).

(6) Suppose that $\Gamma=\{\varphi \Rightarrow(\psi \equiv \chi), \psi\}$. By assumption $\Gamma \vdash \psi$ and Proposition 3.6 part (2), we obtain $\Gamma \vdash \psi \equiv \top$. Using Proposition 3.6 part $(6)$, we get $\Gamma \vdash(\psi \equiv \chi) \equiv(\top \equiv \chi)$. By Proposition 3.6 part (9) and (MP), we have $\Gamma \vdash \varphi \sqcap(\psi \equiv \chi) \equiv \varphi \sqcap(\top \equiv \chi)$. Applying (EL5) and part (1), we obtain $\Gamma \vdash \varphi \sqcap(\psi \equiv \chi) \equiv \varphi \sqcap \chi$. Thus $\vdash \varphi \sqcap \chi \equiv \varphi \sqcap(\psi \equiv \chi)$ by Proposition 3.6 part (4). Using assumption $\Gamma \vdash \varphi \sqcap(\psi \equiv \chi) \equiv \varphi$ and Proposition 3.6 part (7), we have $\Gamma \vdash \varphi \sqcap \chi \equiv \varphi$, that is $\Gamma \vdash \varphi \Rightarrow \chi$.

(7) Let $\Gamma=\{\varphi, \psi\}$. By assumption $\Gamma \vdash \varphi$, Proposition 3.6 part (2), (EL8) and (EA), we have $\Gamma \vdash(\varphi \sqcap \psi) \equiv(\top \sqcap \psi)$. By assumption $\Gamma \vdash \psi$, (EL4) and (EA), we get $\Gamma \vdash \psi \sqcap \top$. Thus $\Gamma \vdash \top \sqcap \psi$ by Proposition 3.6 part (4). By Proposition 3.6 part (10), we get result.

(8) By assumption and Proposition 3.6 part (9), we have $\varphi \equiv \psi \vdash(\varphi \sqcap$ $\varphi) \equiv(\varphi \sqcap \psi)$. Since $\Gamma \vdash((\varphi \sqcap \varphi) \equiv(\varphi \sqcap \psi)) \Rightarrow((\varphi \sqcap \varphi) \equiv \varphi) \equiv$ $(\varphi \sqcap \psi) \equiv \varphi))$ by $($ EL9), then $\Gamma \vdash((\varphi \sqcap \varphi) \equiv \varphi) \equiv((\varphi \sqcap \psi) \equiv \varphi)$. Applying (EL1) and (EA), we have $\Gamma \vdash(\varphi \sqcap \psi) \equiv \varphi$, that is (6) $\varphi \equiv \psi \vdash \varphi \Rightarrow \psi$.

(9) Suppose that $\Gamma=\left\{(\varphi \equiv \psi) \Rightarrow \chi, \varphi \equiv \varphi_{1}\right\}$. By assumption $\Gamma \vdash \varphi \equiv$ $\varphi_{1}$ and Proposition 3.6 part $(6)$, we have $\vdash(\varphi \equiv \psi) \equiv\left(\varphi_{1} \equiv \psi\right)$. Applying (EL8), we obtain $\Gamma \vdash((\varphi \equiv \psi) \sqcap \chi) \equiv\left(\left(\varphi_{1} \equiv \psi\right) \sqcap \chi\right)$. Thus $\Gamma \vdash\left(\left(\varphi_{1} \equiv \psi\right) \sqcap \chi\right) \equiv((\varphi \equiv \psi) \sqcap \chi)$ by Proposition 3.6 part (4). Also, using assumption $\Gamma \vdash((\varphi \equiv \psi) \sqcap \chi) \equiv(\varphi \equiv \psi)$ and twice Proposition 3.6 part $(7)$, we get $\Gamma \vdash\left(\left(\varphi_{1} \equiv \psi\right) \sqcap \chi\right) \equiv\left(\varphi_{1} \equiv \psi\right)$, that is $\Gamma \vdash\left(\varphi_{1} \equiv \psi\right) \Rightarrow \chi$.

Proposition 3.8. Let $\varphi, \psi, \chi \in \mathcal{F}$ be formulas. $\mathcal{E} \mathcal{L}$ proves the following properties of implication:
(1) $\vdash \varphi \Rightarrow \varphi$,
(2) $\vdash(\top \Rightarrow \varphi) \equiv \varphi$,
$(3) \vdash(\varphi \Rightarrow \psi) \Rightarrow((\varphi \sqcap \chi) \Rightarrow \psi)$, 
(4) $\vdash \varphi \Rightarrow(\psi \Rightarrow \varphi)$,

$(5) \vdash \varphi \Rightarrow(\varphi \equiv \top)$,

$(6) \vdash(\varphi \sqcap \psi) \Rightarrow \varphi, \vdash(\varphi \sqcap \psi) \Rightarrow \psi$,

(7) $\{\varphi \Rightarrow \psi, \psi \Rightarrow \chi\} \vdash \varphi \Rightarrow \chi$,

$(8) \vdash(\varphi \Rightarrow \psi) \Rightarrow((\varphi \sqcap \chi) \Rightarrow \psi)$.

\section{PROOF:}

(1) It follows from (EL1).

(2) The proof is straightforward by (EL5), (EL4) and Proposition 3.7 part (1).

(3) We have $\vdash((\varphi \sqcap \psi) \equiv \varphi) \Rightarrow(((\varphi \sqcap \psi) \sqcap \chi) \equiv(\varphi \sqcap \chi))$ by (EL8). Using (EL2), (EL3) and Proposition 3.7 part $(2)$, we obtain $\vdash((\varphi \sqcap \psi) \equiv$ $\varphi) \Rightarrow(((\varphi \sqcap \chi) \sqcap \psi) \equiv(\varphi \sqcap \chi))$. Hence $\vdash(\varphi \Rightarrow \psi) \Rightarrow((\varphi \sqcap \chi) \Rightarrow \psi)$ by definition $\Rightarrow$.

(4) We have $\vdash(\top \Rightarrow \varphi) \Rightarrow((\top \sqcap \psi) \Rightarrow \varphi)$ by part $(3)$. Since $\vdash(\top \Rightarrow \varphi) \equiv$ $\varphi$ by part (2), then $\vdash \varphi \Rightarrow((\top \sqcap \psi) \Rightarrow \varphi)$ by Proposition 3.7 part (5). By definition implication, $\vdash \varphi \Rightarrow(((\top \sqcap \psi) \sqcap \varphi) \equiv(\top \sqcap \psi))$. Using (EL4) and Proposition 3.7 part (4), we obtain $\vdash \varphi \Rightarrow(((\top \sqcap \psi) \sqcap \varphi) \equiv$ $\psi)$. Applying (EL4), (EL8) and (MP), we have $\vdash((\top \sqcap \psi) \sqcap \varphi) \equiv$ $(\psi \sqcap \varphi)$. Hence $\vdash \varphi \Rightarrow(((\psi \sqcap \varphi) \equiv \psi)$ by Proposition 3.7 part (3), that is $\vdash \varphi \Rightarrow(\psi \Rightarrow \varphi)$.

(5) Applying (EL5), (EL8), we have $\vdash(\varphi \sqcap(\varphi \equiv \top)) \equiv(\varphi \sqcap \varphi)$. By (EL1) and Proposition 3.6 part (7), we get $\vdash(\varphi \sqcap(\varphi \equiv \top)) \equiv \varphi$. Hence $\vdash \varphi \Rightarrow(\varphi \equiv \top)$.

(6) By (EL9), We have $\vdash(((\varphi \sqcap \varphi) \sqcap \psi) \equiv(\varphi \sqcap(\varphi \sqcap \psi))) \Rightarrow(((\varphi \sqcap \varphi) \sqcap \psi \equiv$ $(\varphi \sqcap \psi)) \equiv(\varphi \sqcap(\varphi \sqcap \psi) \equiv(\varphi \sqcap \psi)))$. Using (EL3) and (MP), we get $\vdash((\varphi \sqcap \varphi) \sqcap \psi \equiv(\varphi \sqcap \psi)) \equiv(\varphi \sqcap(\varphi \sqcap \psi) \equiv(\varphi \sqcap \psi))$. We have $\vdash(\varphi \sqcap \varphi) \sqcap \psi \equiv(\varphi \sqcap \psi)$ by (EL1), (EL8) and (MP). Thus $\vdash \varphi \sqcap(\varphi \sqcap \psi) \equiv(\varphi \sqcap \psi)$ by (EA). Hence $\vdash(\varphi \sqcap \psi) \Rightarrow \varphi$ by definition of implication. 
(7) By assumptions, definition of implication and Proposition 3.6 part (4), we have $\vdash(\psi \sqcap \varphi) \equiv \varphi$ and $\vdash \psi \equiv(\psi \sqcap \chi)$. Using Proposition 3.7 part (1), we obtain $\vdash((\psi \sqcap \chi) \sqcap \varphi) \equiv \varphi$. By Proposition 3.6 part (5), $\vdash \varphi \sqcap(\psi \sqcap \chi) \equiv \varphi$. Hence we get the result by (EL7) and (MP).

(8) It follows from (EL8) and definition $\Rightarrow$.

In the following, we will use the standard Lindenbaum Tarski technique to show that propositional equality logic $\mathcal{E} \mathcal{L}$.

Lemma 3.9. Let $\varphi_{1}, \varphi_{2}, \psi_{1}, \psi_{2} \in \mathcal{F}$ be formulas. $\mathcal{E} \mathcal{L}$ proves the following properties:

(1) $\left\{\varphi_{1} \equiv \psi_{1}, \varphi_{2} \equiv \psi_{2}\right\} \vdash\left(\varphi_{1} \sqcap \varphi_{2}\right) \equiv\left(\psi_{1} \sqcap \psi_{2}\right)$,

(2) $\left\{\varphi_{1} \equiv \psi_{1}, \varphi_{2} \equiv \psi_{2}\right\} \vdash\left(\varphi_{1} \equiv \varphi_{2}\right) \equiv\left(\psi_{1} \equiv \psi_{2}\right)$.

Proof: Suppose that $\Gamma=\left\{\varphi_{1} \equiv \psi_{1}, \varphi_{2} \equiv \psi_{2}\right\}$.

(1) By assumption $\Gamma \vdash \varphi_{1} \equiv \psi_{1}$, (EL8) and (MP), we have $\Gamma \vdash\left(\varphi_{1} \sqcap\right.$ $\left.\varphi_{2}\right) \equiv\left(\psi_{1} \sqcap \varphi_{2}\right)$. By Proposition 3.6 part $(9)$ we obtan $\Gamma \vdash\left(\psi_{1} \sqcap \varphi_{2}\right) \equiv$ $\left(\psi_{1} \sqcap \psi_{2}\right)$. By Proposition 3.6 part $(7)$, we get $\Gamma \vdash\left(\varphi_{1} \sqcap \varphi_{2}\right) \equiv$ $\left(\psi_{1} \sqcap \psi_{2}\right)$.

(2) Using assumption $\Gamma \vdash \varphi_{1} \equiv \psi_{1}$, (EL9) and (MP), we have $\Gamma \vdash\left(\varphi_{1} \equiv\right.$ $\left.\varphi_{2}\right) \equiv\left(\psi_{1} \equiv \varphi_{2}\right)$. Applying assumption $\Gamma \vdash \varphi_{2} \equiv \psi_{2}$, Proposition 3.6 part (8) and (MP), we obtain $\Gamma \vdash\left(\psi_{1} \equiv \varphi_{2}\right) \equiv\left(\psi_{1} \equiv \psi_{2}\right)$. Therefore $\Gamma \vdash\left(\varphi_{1} \equiv \varphi_{2}\right) \equiv\left(\psi_{1} \equiv \psi_{2}\right)$ by Proposition 3.6 part $(7)$.

Proposition 3.10. Let $\Gamma$ be a theory over the propositional equality logic $\mathcal{E} \mathcal{L}$. Put $\varphi \approx_{\Gamma} \psi$ iff $\Gamma \vdash \varphi \equiv \psi$. Then $\approx_{\Gamma}$ is an equivalence relation on $\mathcal{F}$.

PROOF: It follows from Proposition 3.6 part (1), part (4) and part (7) that $\approx_{\Gamma}$ is an equivalence on relation on $\mathcal{F}$.

Let $\Gamma$ be a theory over the propositional equality logic $\mathcal{E} \mathcal{L}$. Denote $M_{\Gamma}=$ $\left\{[\varphi]_{\Gamma}: \varphi \in \mathcal{F}\right\}$ where $[\varphi]_{\Gamma}=\left\{\psi \in \mathcal{F} \mid \varphi \approx_{\Gamma} \psi\right\}$. Finally, we define

$[\varphi]_{\Gamma} \wedge[\psi]_{\Gamma}=[\varphi \sqcap \psi]_{\Gamma}$,

$[\varphi]_{\Gamma} \sim[\psi]_{\Gamma}=[\varphi \equiv \psi]_{\Gamma}$,

$1=[\top]_{\Gamma}$. 
Proposition 3.11. The algebra $\mathcal{M}_{\Gamma}=\left(M_{\Gamma}, \wedge, \sim, 1\right)$ is an equality-algebra. Proof: By Lemma 3.9, we know that the operations $\wedge$ and $\sim$ are well defined. By $\left(\right.$ EL1) $-\left(\right.$ EL4), we can see that $\left(M_{\Gamma}, \wedge, \sim, 1\right)$ i a meet-semilattice with top element 1 . Now, we will show that $[\varphi]_{\Gamma} \leq[\psi]_{\Gamma}$ iff $T \vdash \varphi \Rightarrow \psi$. Suppose that $\Gamma \vdash \varphi \Rightarrow \psi$. Then $\Gamma \vdash(\varphi \sqcap \psi) \equiv \varphi$. So $[\varphi]_{\Gamma} \wedge[\psi]_{\Gamma}=[\varphi]_{\Gamma}$. Hence $[\varphi]_{\Gamma} \leq[\psi]_{\Gamma}$. Similarly, we can prove if $[\varphi]_{\Gamma} \leq[\psi]_{\Gamma}$, then $\Gamma \vdash \varphi \Rightarrow \psi$. The proof of (EL5)-(EL9) is straightforward.

TheOrem 3.12 (Completeness). The propositional equality logic $\mathcal{E} \mathcal{L}$ is complete, i.e. the following are equivalent:

(i) $\vdash \varphi$,

(ii) for every equality-algebra $\mathcal{A}=(A, \wedge, \sim, 1), \varphi$ is an $\mathcal{A}$-tautology.

Proof: (i) $\Rightarrow$ (ii) follows from Theorem 3.5. Conversely, for every theory $\Gamma$ of the propositional equality logic $\mathcal{E} \mathcal{L}, \mathcal{M}_{\Gamma}=\left(M_{T}, \wedge, \sim, 1\right)$ is an equalityalgebra. Let $\Gamma$ to be the set of all axioms of $\mathcal{E} \mathcal{L}$. Thus $\varphi$ is an $\mathcal{M}_{\Gamma^{-}}$ tautology by assumption. Consider the mapping $e$ defined by $e(p)=[p]_{\Gamma}$ for all propositional variables $p$. Then $e$ is a $\mathcal{M}$-evaluation from $\mathcal{F}$ to the equality-algebra $\mathcal{M}$. By Definition 3.2, $e(\varphi)=[1]_{\Gamma}$. Then $[\varphi]_{\Gamma}=[1]_{\Gamma}$, that is $\Gamma \vdash \varphi \equiv \top$. Hence $\vdash \varphi$.

Now, we will show the locally deduction theorem for the propositional equality logic $\mathcal{E} \mathcal{L}$. For this, we need the following proposition.

For convenience, we shall abbreviate the formulas $\varphi \equiv(\cdots \equiv(\varphi \equiv$ $\psi) \cdots)$ and $\varphi \Rightarrow(\cdots \Rightarrow(\varphi \Rightarrow \psi) \cdots)$ by $\varphi \equiv^{n} \psi$ and $\varphi \Rightarrow^{n} \psi, n \in \mathbb{N}_{0}$ indicating the number of occurrences of $\varphi$.

Proposition 3.13. Let $\varphi, \psi, \chi \in \mathcal{F}$ be formulas. $\mathcal{E} \mathcal{L}$ proves the following:

(1) $\varphi \Rightarrow \psi \vdash(\chi \Rightarrow \varphi) \Rightarrow(\chi \Rightarrow \psi)$,

(2) $\varphi \Rightarrow \psi \vdash(\psi \Rightarrow \chi) \Rightarrow(\varphi \Rightarrow \chi)$,

(3) $\vdash \varphi \Rightarrow[(\psi \equiv \varphi) \equiv \psi]$,

(4) $\vdash(\varphi \equiv \psi) \Rightarrow(\varphi \Rightarrow \psi)$,

$(5) \vdash \varphi \Rightarrow((\varphi \Rightarrow \psi) \Rightarrow \psi)$,

$(6) \vdash(\varphi \Rightarrow \psi) \Rightarrow[(\psi \Rightarrow \chi) \Rightarrow(\varphi \Rightarrow \chi)]$,

$(7) \vdash(\chi \Rightarrow(\varphi \Rightarrow \psi)) \Rightarrow(\varphi \Rightarrow(\chi \Rightarrow \psi))$, 
$(8) \vdash\left(\varphi \Rightarrow^{n}(\psi \Rightarrow \chi)\right) \Rightarrow\left(\psi \Rightarrow\left(\varphi \Rightarrow^{n} \chi\right)\right)$,

$(9) \vdash(\varphi \Rightarrow \psi) \Rightarrow((\psi \Rightarrow \varphi) \Rightarrow(\varphi \equiv \psi))$,

(10) $\{\varphi \Rightarrow \chi, \varphi \Rightarrow \psi\} \vdash \varphi \Rightarrow(\psi \sqcap \chi)$,

$(11) \vdash(\varphi \equiv \psi) \Rightarrow((\varphi \Rightarrow \psi) \sqcap(\psi \Rightarrow \varphi))$.

\section{PROOF:}

(1) Let $\Gamma=\{\varphi \Rightarrow \psi\}$. Applying (EL7) and definition $\Rightarrow$, we obtain $\vdash((\varphi \sqcap \psi \sqcap \chi) \equiv \chi) \Rightarrow(\chi \Rightarrow \psi)$. By assumption, (EL8) and (MP), we get $\Gamma \vdash(\varphi \sqcap \psi \sqcap \chi) \equiv \varphi \sqcap \chi$. By Proposition 3.7 part (9), we have $\Gamma \vdash((\varphi \sqcap \chi) \equiv \chi) \Rightarrow(\chi \Rightarrow \psi)$, that is $\Gamma \vdash(\chi \Rightarrow \varphi) \Rightarrow(\chi \Rightarrow \psi)$.

(2) Let $\Gamma=\{\varphi \Rightarrow \psi\}$. We have $\vdash((\psi \sqcap \chi) \equiv \psi) \Rightarrow((\varphi \sqcap(\psi \sqcap \chi)) \equiv(\varphi \sqcap \psi))$ by (EL8)and $\Gamma \vdash(\varphi \sqcap \psi) \equiv \varphi$ by assumption. Using Proposition 3.7 part (4), we get $\Gamma \vdash((\psi \sqcap \chi) \equiv \psi) \Rightarrow((\varphi \sqcap \psi \sqcap \chi) \equiv \varphi)$. By (EL7) and Proposition 3.8 part $(7)$, we obtain $\Gamma \vdash((\psi \sqcap \chi) \equiv \psi) \Rightarrow((\varphi \sqcap \chi) \equiv \varphi)$. Hence $\Gamma \vdash(\psi \Rightarrow \chi) \Rightarrow(\varphi \Rightarrow \chi)$.

(3) We have $\vdash(\varphi \equiv \top) \Rightarrow((\varphi \equiv \psi) \equiv(\top \equiv \psi))$ and $\vdash(\psi \equiv \top) \equiv \psi)$ by (EL9) and (EL5) respectively. Therefore $\vdash(\varphi \equiv \top) \Rightarrow((\psi \equiv \varphi) \equiv \psi)$ by Proposition 3.7 part (4). Again by (EL5) and Proposition 3.7 part (5), we get $\vdash \varphi \Rightarrow[(\psi \equiv \varphi) \equiv \psi]$.

(4) Applying (EL8), (EL1) and Proposition 3.7 part (4), we have $\vdash(\varphi \equiv$ $\psi) \Rightarrow((\varphi \sqcap \psi) \equiv \psi)$, that is $\vdash(\varphi \equiv \psi) \Rightarrow(\varphi \Rightarrow \psi)$.

(5) By part (3) and definition $\Rightarrow$, we have $\vdash \varphi \Rightarrow((\varphi \Rightarrow \psi) \equiv(\varphi \sqcap \psi))$. By part (4) and Proposition 3.8 part (7), we get $\vdash \varphi \Rightarrow((\varphi \Rightarrow \psi) \Rightarrow$ $(\varphi \sqcap \psi))$. By Proposition 3.8 part (6) and then part (1), we obtain $\vdash((\varphi \Rightarrow \psi) \Rightarrow(\varphi \sqcap \psi)) \Rightarrow((\varphi \Rightarrow \psi) \Rightarrow \psi)$. Using Proposition 3.8 part (7), we obtain the result.

(6) By (EL8) and part (2), we have $\vdash[((\varphi \sqcap \psi) \equiv(\varphi \sqcap \psi \sqcap \chi)) \Rightarrow(\varphi \equiv$ $(\varphi \sqcap \psi \sqcap \chi))] \Rightarrow[(\psi \sqcap \chi) \equiv \psi) \Rightarrow(\varphi \equiv(\varphi \sqcap \psi \sqcap \chi))]$. By (EL7) and part (1), we have $\vdash[((\psi \sqcap \chi) \equiv \psi) \Rightarrow(\varphi \equiv(\varphi \sqcap \psi \sqcap \chi))] \Rightarrow[((\psi \sqcap \chi) \equiv \psi) \Rightarrow$ $((\varphi \sqcap \chi) \equiv \chi)]$. By (EL9), we have $\vdash((\varphi \sqcap \psi) \equiv \varphi) \Rightarrow[((\varphi \sqcap \psi) \equiv$ $(\varphi \sqcap \psi \sqcap \chi)) \Rightarrow((\varphi \sqcap \psi \sqcap \chi)) \equiv \varphi]$. Using Proposition 3.8 part $(7)$ twice, we obtain $\vdash((\varphi \sqcap \psi) \equiv \varphi) \Rightarrow[((\psi \sqcap \chi) \equiv \psi) \Rightarrow((\varphi \sqcap \chi) \equiv \chi)]$. Hence $\vdash(\varphi \Rightarrow \psi) \Rightarrow[(\psi \Rightarrow \chi) \Rightarrow(\varphi \Rightarrow \chi)]$. 
(7) By part (7), we have $\vdash(\chi \Rightarrow(\varphi \Rightarrow \psi)) \Rightarrow[((\varphi \Rightarrow \psi) \Rightarrow \psi) \Rightarrow(\chi \Rightarrow$ $\psi)]$. By part (5) and then part (2), we have $\vdash[((\varphi \Rightarrow \psi) \Rightarrow \psi) \Rightarrow$ $(\chi \Rightarrow \psi)] \Rightarrow[\varphi \Rightarrow(\chi \Rightarrow \psi)]$. Using Proposition 3.8 part $(6)$, we obtain the result.

(8) It can be proved by part (7) and induction.

(9) It follows from (EL9) and part (4).

(10) Let $\Gamma=\{\varphi \Rightarrow \chi, \varphi \Rightarrow \psi\}$. By assumption $\Gamma \vdash \varphi \Rightarrow \chi$, (EL8) and (MP), we get $\Gamma \vdash((\varphi \sqcap \chi) \sqcap \psi) \equiv(\varphi \sqcap \psi)$. Using assumption $\Gamma \vdash \varphi \Rightarrow \psi$ and (EL9), we obtain the result.

(11) It follows from part (4) and part (10).

TheOREM 3.14 (Local Deduction Theorem). Let $\Gamma$ be a theory over the propositional equality logic $\mathcal{E} \mathcal{L}$ and $\varphi, \psi$ be formulas. Then $\Gamma \cup\{\varphi\} \vdash \psi$ if and only if $\Gamma \vdash \varphi \Rightarrow^{n} \psi$ where $n \in \mathbb{N}_{0}$.

Proof: Suppose that $\Gamma \cup\{\varphi\} \vdash \psi$. We will prove it by induction on the number of formulas on the sequence of deduction of $\psi$ from $\Gamma \cup\{\varphi\}$. Let $\chi_{1}, \chi_{2}, \ldots, \chi_{k}$ be a corresponding $\Gamma \cup\{\varphi\}$-proof of $\psi$. We should consider four cases:

Case 1: $\psi$ is an axiom of $\mathcal{E} \mathcal{L}$ or $\psi \in \Gamma$. By Proposition 3.8 part (4) and (MP), we obtain $\Gamma \vdash \varphi \Rightarrow \psi$.

Case 2: $\psi$ is $\varphi$. By Proposition 3.8 part (1), we have $\Gamma \vdash \varphi \Rightarrow \varphi$.

Case 3: $\psi$ is obtained from two pervious formulas on the corresponding $\Gamma \cup\{\varphi\}$-proof of $\psi$ by an application (MP). These two formulas must have the form $\chi_{i}$ and $\chi_{i} \Rightarrow \psi$ where $1<i<k$. By the induction hypothesis, there exist $n, m \in \mathbb{N}_{0}$ such that $T \vdash \varphi \Rightarrow^{n} \chi_{i}$ and $\Gamma \vdash$ $\varphi \Rightarrow^{m}\left(\chi_{i} \Rightarrow \psi\right)$.

By Proposition 3.13 part (8) and (MP), we get $\Gamma \vdash \chi_{i} \Rightarrow\left(\varphi \Rightarrow^{m} \chi\right)$. Using Proposition 3.13 part (1), we have $\Gamma \vdash\left(\varphi \Rightarrow^{n} \chi_{i}\right) \Rightarrow\left(\varphi \Rightarrow^{n}\right.$ $\left.\left(\varphi \Rightarrow^{m} \psi\right)\right)$. Applying (MP), we obtain $\Gamma \vdash \varphi \Rightarrow^{n}\left(\varphi \Rightarrow^{m} \psi\right)$. Hence $\Gamma \vdash \varphi \Rightarrow^{n+m} \psi$.

Case 4: $\psi$ results by (EA) from pervious member $\chi_{i}$ and $\chi_{i} \equiv \psi(1<i<k)$ of the corresponding $\Gamma \cup\{\varphi\}$-proof of $\psi$. Thus $\Gamma \cup\{\varphi\} \vdash \chi_{i}$ and $T \cup\{\varphi\} \vdash$ 
$\chi_{i} \equiv \psi$. By Proposition 3.7 part (8), we have $\Gamma \cup\{\varphi\} \vdash \chi_{i} \Rightarrow \psi$. As Case 3 above, we can show that $\Gamma \vdash \varphi \Rightarrow^{n+m} \psi$.

Conversely, suppose that $\Gamma \vdash \varphi \Rightarrow^{n} \psi$ for $n>1$. Then $\Gamma \vdash \varphi \Rightarrow\left(\varphi \Rightarrow^{n-1}\right.$ $\psi)$. Thus $\Gamma \cup\{\varphi\} \vdash \varphi \Rightarrow^{n-1} \psi$. Replacing this, we obtain $\Gamma \cup\{\varphi\} \vdash \varphi \Rightarrow \psi$. Hence $\Gamma \cup\{\varphi\} \vdash \psi$.

Remark. The deduction theorem in the form of $\Gamma \cup\{\varphi\} \vdash \psi$ if and only if $\Gamma \vdash$ $\varphi \Rightarrow \psi$ does not hold in the propositional equality logic $\mathcal{E} \mathcal{L}$. Suppose that it holds and $\varphi \in \mathcal{F}$ be arbitrary formula. Then $\{\varphi, \varphi \Rightarrow(\varphi \Rightarrow \psi)\} \vdash \varphi$. Hence $\vdash(\varphi \Rightarrow(\varphi \Rightarrow \psi)) \Rightarrow(\varphi \Rightarrow \psi)$. Therefore $(\varphi \Rightarrow(\varphi \Rightarrow \psi)) \Rightarrow(\varphi \Rightarrow \psi)$ is an $\mathcal{A}$-tautology for every equality-algebra $\mathcal{A}=(A, \exists, \forall)$ by Theorem 3.12. Now, consider equality-algebra in Example 4.7 and define $e(\varphi)=a$ and $e(\psi)=b$. Then $(e(\varphi) \rightarrow(e(\varphi) \rightarrow e(\psi))) \rightarrow(e(\varphi) \rightarrow e(\psi))=1 \rightarrow d=d$ which is a contradiction.

In the following, we will show that the propositional equality logic $\mathcal{E} \mathcal{L}$ algebraizable with respect to the variety of equality-algebras in the sense of [1] (Also see [2]).

TheOREM 3.15. The propositional equality logic $\mathcal{E} \mathcal{L}$ is algebraizable with the defining equation $\varphi=\top$ and the equivalence formulas $\{\varphi \equiv \psi\}$.

Proof: Suppose that $\varphi \Delta \psi=\{\varphi \equiv \psi\}, \delta(\varphi)=\varphi$ and $\epsilon(\varphi)=\top$. By the intrinsic characterization given by Blok and Pigozzi [1, Theorem 4.7], it is sufficient to check that the following conditions hold for all formulas:

(1) $\vdash \varphi \Delta \varphi$,

(2) $\varphi \Delta \psi \vdash \psi \Delta \varphi$,

(3) $\varphi \Delta \psi, \psi \Delta \chi \vdash \varphi \Delta \chi$,

(4) $\varphi_{1} \Delta \psi_{1}, \varphi_{2} \Delta \psi_{2} \vdash\left(\varphi_{1} \sqcap \varphi_{2}\right) \Delta\left(\psi_{1} \sqcap \psi_{2}\right)$,

(5) $\varphi_{1} \Delta \psi_{1}, \varphi_{2} \Delta \psi_{2} \vdash\left(\varphi_{1} \equiv \varphi_{2}\right) \Delta\left(\psi_{1} \equiv \psi_{2}\right)$,

(6) $\varphi \dashv \Vdash \delta(\varphi) \Delta \epsilon(\varphi)$.

Now, we will prove them as follows:

(1) Since $\varphi \Delta \varphi=\{\varphi \equiv \varphi\}$, then $\vdash \varphi \Delta \varphi$ by Proposition 3.6 part (1). 
(2) By Proposition 3.6 part (4), $\varphi \equiv \psi \vdash \psi \equiv \varphi$. Hence $\varphi \Delta \psi \vdash \psi \Delta \varphi$.

(3) It follows from Proposition 3.6 part (7).

(4) and (5) We obtain them by Lemma 3.9.

(6) Applying Proposition 3.6 part (2) and part (3), we have $\varphi \dashv \varphi \equiv \top$. Hence $\varphi \dashv-\delta(\varphi) \Delta \epsilon(\varphi)$.

THEOREM 3.16. The propositional equality logic $\mathcal{E} \mathcal{L}$ is algebraizable with respect to the variety of equality-algebras, with equivalence formulas $\{\varphi \equiv$ $\psi\}$ and defining equation $\varphi=T$.

Proof: Let $A l g^{*} \mathcal{E} \mathcal{L}$ be the algebraic semantics of the propositional equality logic $\mathcal{E} \mathcal{L}$. By Theorem 3.15, it exists and we can take $\{\varphi \equiv \psi\}$ for the equivalence formulas, and $\delta(p)=p, \epsilon(p)=\top$ for the defining equation. By [1, Theorem 2.17], the variety $A l g^{*} \mathcal{E} \mathcal{L}$ is axiomatized as follows:

(1) $(x \wedge x) \sim x=1$.

(2) $(x \wedge y) \sim(y \wedge x)=1$,

(3) $((x \wedge y) \wedge z) \sim(x \wedge(y \wedge z))=1$.

(4) $((x \wedge 1) \sim x)=1$,

(5) $(x \sim 1) \sim x=1$,

(6) $(x \sim y) \sim(y \sim x)=1$,

(7) $((x \wedge y \wedge z) \sim x) \rightarrow((x \wedge y) \sim x)=1$,

(8) $(x \sim y) \rightarrow((x \wedge z) \sim(y \wedge z))=1$,

(9) $(x \sim y) \rightarrow((x \sim z) \sim(y \sim z))=1$,

(10) $x=1$ and $x \sim y=1$ imply $y=1$,

(11) $x=1$ and $x \rightarrow y=1$ imply $y=1$,

(12) $x \sim y=1$ imply $x=y$.

It is obvious that every equality-algebra satisfies (1)-(12). Hence the variety of equality-algebras is included in $A l g^{*} \mathcal{E} \mathcal{L}$. Conversely, let 
$\mathcal{A}=(A, \wedge, \sim, 1)$ be an algebra belonging to $A l g^{*} \mathcal{E} \mathcal{L}$. Then $(A, \wedge, 1)$ is a meet-semilattice with top element 1 by part (1)-(5) and part (12). (E5) follows from part (7) and Proposition 2.2. It is clear that $\mathcal{A}$ satisfies the other conditions of Definition 2.1.

Therefore We conclude that $A l g^{*} \mathcal{E} \mathcal{L}$ is precisely the variety of all equality-algebras.

In 1990, Skolem semilattices were defined by Büchi and Owens (see [5]). Let $x, y$ be arbitrary elements of a meet-semilattice $(S, \wedge, 1)$ with the greatest element 1. If the largest element of the set $\{z \in S: a \wedge x=b \wedge x\}$ exists, then it is called the symmetric relative pseudo-complement (the symmetric $\mathrm{RPC}$ ) of $x$ and $y$, and is denoted by $x \leftrightarrow_{s} y$. If the the symmetric RPC exists for every pair of elements $x, y$, then the enriched structure $\left(S, \wedge, \leftrightarrow_{s}, 1\right)$ is called a Skolem semilattice.

The class of Skolem semilattices is a strongly point-regular and forms a Hilbertian variety and hence Fregean. Skolem semilattices form the algebraic semantics of the conjunctive-equivalential fragment of intuitionistic $\operatorname{logic}([7])$.

Proposition 3.17. The Skolem semilattices form a proper subvariety of the variety of the equality algebras.

Proof: Let $x, y$ be arbitrary elements of the Skolem semilattice $\left(S, \wedge, \leftrightarrow_{s}, 1\right)$. We define $x \sim y:=x \leftrightarrow_{s} y$. Then (E2)-(E4) hold by parts (1)-(3) of Theorem 6.5.2 in [7]. The proof of (E5) and (E6) is easy. Let $t \in S$ such that $t \wedge x=t \wedge x$. By part of (4) of Theorem 6.5.2 in [7], we have $t \wedge\left(x \leftrightarrow_{s} z\right)=t \wedge\left((x \wedge t) \leftrightarrow_{s} z\right)$. Thus $t \wedge(x \sim z)=t \wedge((x \wedge t) \sim z)=t \wedge((y \wedge t) \sim z)=t \wedge(y \sim z)$. We obtain $t \leq \sup \{w \in S: w \wedge(x \sim z)=w \wedge(x \sim z)\}=(x \sim z) \sim(y \sim z)$. Then (E7) hold. Hence $(S, \wedge, \sim, 1)$ is an equality algebra. It follows that Skolem semilattices form a subvariety of the variety of the equality algebras. This inclusion is proper, because the logic determined by Skolem semilattice admits the standard deduction theorem while the logic determined by equality algebras admits merely a local deduction theorem by Theorem 3.14.

Corollary 3.18. The propositional equality logic $\mathcal{E} \mathcal{L}$ with respect to the variety of equality-algebras is regularly algebraizable but it is not Fregean.

Proof: Let $E(\varphi, \psi):=\{\varphi \equiv \psi\}$. Then $E(\varphi, \psi)$ is a (finite) system of equivalence sentences for $\mathcal{E} \mathcal{L}$ and the G-rule determined by $E$ is valid in 
$\mathcal{E} \mathcal{L}$. Thus $\mathcal{E} \mathcal{L}$ is finitely regularly algebraizable. By Corollary 6.5.11 in [7] and Theorem 3.15, we conclude that $\mathcal{E} \mathcal{L}$ is not Fregean.

In 1966, famous Polish logician Roman Suszko create a new logical calculus called by him Non-Fregean Logic (see [20]). He introduced the identity connective to metalogic and, relying on Wittgenstein's writings, he has initiated systematic investigations of deductive systems endowed with identity. By the above corollary, the equality algebras are the algebraic counterparts of a strengthening of the pure Suszko logic with identity and additionally equipped with the connective that possesses the properties of conjunction.

\section{Equality $_{\triangle}$-algebras}

In this section, the concept of (prelinear) equality $\triangle$-algebra is introduced and some related properties are investigated.

DeFinition 4.1. An equality $\triangle$-algebra is an algebra $(A, \wedge, \sim, \triangle, 0,1)$ of type $(2,2,1,0,0)$ where $(A, \wedge, \sim, 0,1)$ is a bounded equality-algebra expanded by a unary operation $\triangle: A \rightarrow A$ satisfying the following:

$(\triangle 1) \triangle 1=1$,

$(\triangle 2) \triangle x \leq x$,

$(\triangle 3) \triangle x \leq \triangle \triangle x$

$(\triangle 4) \triangle(x \sim y) \leq \triangle x \sim \triangle y$,

$(\triangle 5) \triangle(x \wedge y)=\triangle x \wedge \triangle y$,

$(\triangle 6)$ if $x \vee y$ and $\triangle x \vee \triangle y$ exist, then $\triangle(x \vee y) \leq \triangle x \vee \triangle y$,

( $\triangle 7) \triangle x \vee \neg \triangle x=1$, that is 1 is unique upper bound of the set $\{\triangle x, \neg \triangle x\}$ in $A$.

Example 4.2.

(1) Let $(A, \wedge, \sim, 0,1)$ be a bounded equality-algebra. Define $\triangle: A \rightarrow A$ by $\triangle 1=1$ and $\triangle x=0$ for any $x<0$. Then $(A, \wedge, \sim, \triangle, 0,1)$ is an equality $\triangle$-algebra.

(2) Let $A=\{0, a, b, c, d, 1\}$ be a lattice in Fig. 1. Consider the operations $\sim$ and $\rightarrow$ given by the following tables: 


\begin{tabular}{|c|c|c|c|c|c|c|c|c|c|c|c|c|c|}
\hline$\sim$ & 0 & $a$ & $b$ & $c$ & $d$ & 1 & $\rightarrow$ & 0 & $a$ & $b$ & $c$ & $d$ & 1 \\
\hline 0 & 1 & $d$ & $c$ & $b$ & $a$ & 0 & 0 & 1 & 1 & 1 & 1 & 1 & 1 \\
\hline$a$ & $d$ & 1 & $a$ & $d$ & $c$ & $a$ & $a$ & $d$ & 1 & $a$ & $c$ & $c$ & 1 \\
\hline$b$ & $c$ & $a$ & 1 & 0 & $d$ & $b$ & $b$ & $c$ & 1 & 1 & $c$ & $c$ & 1 \\
\hline$c$ & $b$ & $d$ & 0 & 1 & $a$ & $c$ & $c$ & $b$ & $a$ & $b$ & 1 & $a$ & 1 \\
\hline$d$ & $a$ & $c$ & $d$ & $a$ & 1 & $d$ & $d$ & $a$ & 1 & $a$ & 1 & 1 & 1 \\
\hline 1 & 1 & 1 & 1 & 1 & 1 & 1 & 1 & 0 & $a$ & $b$ & $c$ & $d$ & 1 \\
\hline
\end{tabular}

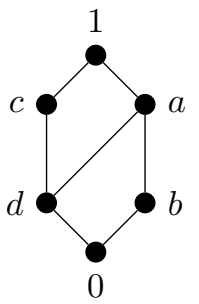

Figure 1

Then $\mathcal{A}=(A, \wedge, \sim, 0,1)$ is a bounded equality-algebra ([22]). Define the unary operation $\triangle$ on $A$ as $\triangle 0=\triangle d=0, \triangle a=\triangle b=b, \triangle c=c$ and $\triangle 1=1$. Then $(A, \wedge, \sim, \triangle, 0,1)$ is an equality $\triangle$-algebra.

Remark 4.3. It is obvious that every equality $\triangle$-algebra is a true equality algebra. But the converse may not be true in general. Consider the following example:

Example 4.4. Let $A=\{0, a, b, 1\}$ be a chain such that $0<a<b<1$. Consider the operations $\sim$ and $\rightarrow$ given by the following tables:

\begin{tabular}{c|cccc}
$\sim$ & 0 & $a$ & $b$ & 1 \\
\hline 0 & 1 & $a$ & $a$ & 0 \\
$a$ & 1 & 1 & $a$ & $a$ \\
$b$ & 1 & 1 & 1 & $b$ \\
1 & 1 & 1 & 1 & 1
\end{tabular}

\begin{tabular}{r|cccc}
$\rightarrow$ & 0 & $a$ & $b$ & 1 \\
\hline 0 & 1 & 1 & 1 & 1 \\
$a$ & $a$ & 1 & 1 & 1 \\
$b$ & 0 & $a$ & 1 & 1 \\
1 & 0 & $a$ & $b$ & 1
\end{tabular}

Then $\mathcal{A}=(A, \wedge, \sim, 0,1)$ is a bounded equality-algebra ([8]). Define the unary operation $\tau$ on $A$ as follows:

$$
\tau(0)=0, \tau(a)=\tau(b)=a \text { and } \tau(1)=1 .
$$


Then $\tau$ is a very true operation on $A([21])$. Since $\tau(a) \vee \neg \tau(a)=a \neq 1$, then $\tau$ is not $(A, \wedge, \sim, \tau, 0,1)$ is not an equality $\triangle$-algebra.

Proposition 4.5. Let $(A, \wedge, \sim, \triangle, 0,1)$ be an equality $\triangle$-algebra. Then the following properties hold, for all $x, y, z \in A$ :

(1) $\triangle x=1$ if and only if $x=1$,

(2) $x \leq y$ implies $\triangle x \leq \triangle y$,

(3) $\triangle \triangle x=\triangle x$,

(4) $\triangle x \leq y$ if and only if $\triangle x \leq \triangle y$,

(5) $\operatorname{Im}(\triangle)=\operatorname{Fix}(\triangle)$ where $\operatorname{Fix}(\triangle)=\{x \in A: \triangle x=x\}$,

(6) if $\triangle$ is surjective, then $\triangle=I d_{A}$,

(7) $\operatorname{Ker}(\triangle)=\{1\}$, where $\operatorname{Ker}(\triangle)=\{x \in A: \triangle x=1\}$,

(8) $\operatorname{Ker}(\triangle)$ is a deductive system of $A$,

(9) $\triangle(x \rightarrow y) \leq \triangle x \rightarrow \triangle y$,

(10) if $x \vee y$ and $\triangle x \vee \triangle y$ exist, then $\triangle(x \vee y)=\triangle x \vee \triangle y$.

Proof: Since every equality $\triangle$-algebra is a very true equality-algebra, then part (1)-(9) follow from Proposition in [21]. (10) follows from $(\triangle 6)$ and part (2).

DeFINITION 4.6. A prelinear equality $\triangle$-algebra is an equality $\triangle$-algebra $(A, \wedge, \sim, \triangle, 0,1)$ satisfies the following: for all $x, y, z \in A$

$(\triangle 8) \triangle(x \rightarrow y) \rightarrow z \leq(\triangle(y \rightarrow x) \rightarrow z) \rightarrow z$.

Example 4.7.

(1) An equality $\triangle$-algebra in Example 4.2 part (2) is a prelinear equality $\triangle^{-}$ algebra.

(2) Let $A=\{0, a, b, c, d, 1\}$ be a lattice in Fig. 2. Consider the operations $\sim$ and $\rightarrow$ given by the following tables: 


\begin{tabular}{c|llllll}
$\sim$ & 0 & $a$ & $b$ & $c$ & $d$ & 1 \\
\hline 0 & 1 & $d$ & $d$ & $d$ & $c$ & 0 \\
$a$ & $d$ & 1 & $c$ & $d$ & $c$ & $a$ \\
$b$ & $d$ & $c$ & 1 & $d$ & $c$ & $b$ \\
$c$ & $d$ & $d$ & $d$ & 1 & $d$ & $c$ \\
$d$ & $c$ & $c$ & $c$ & $d$ & 1 & $d$ \\
1 & 0 & $a$ & $b$ & $c$ & $d$ & 1
\end{tabular}

\begin{tabular}{c|cccccc}
$\rightarrow$ & 0 & $a$ & $b$ & $c$ & $d$ & 1 \\
\hline 0 & 1 & 1 & 1 & 1 & 1 & 1 \\
$a$ & $d$ & 1 & $d$ & 1 & 1 & 1 \\
$b$ & $d$ & $d$ & 1 & 1 & 1 & 1 \\
$c$ & $d$ & $d$ & $d$ & 1 & 1 & 1 \\
$d$ & $c$ & $c$ & $c$ & $d$ & 1 & 1 \\
1 & 0 & $a$ & $b$ & $c$ & $d$ & 1
\end{tabular}

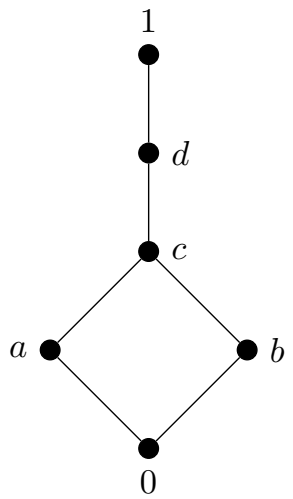

Figure 2

Then $\mathcal{A}=(A, \wedge, \sim, 0,1)$ is a bounded equality-algebra ([22]). Define the unary operation $\triangle$ on $A$ as $\triangle 0=\triangle a=\triangle b=\triangle c=\triangle d=0$ and $\triangle 1=1$. Then $(A, \wedge, \sim, \triangle, 0,1)$ is an equality $\triangle$-algebra but it is not a prelinear equality $\triangle$-algebra because $\triangle(a \rightarrow b) \rightarrow c=1 \not \leq c=$ $(\triangle(b \rightarrow y) \rightarrow c) \rightarrow c$.

(3) Let $(A, \wedge, \sim, 0,1)$ be a prelinear bounded equality-algebra. Define $\triangle: A \rightarrow A$ by $\triangle 1=1$ and $\triangle x=0$ for any $x<0$. Then $(A, \wedge, \sim$ $, \triangle, 0,1)$ is a prelinear equality $\triangle$-algebra.

The proof of the following proposition is similar to Lemma 8 in [8].

Proposition 4.8. Let $\triangle$ be a unary operation on a bounded equalityalgebra $\mathcal{A}=(A, \wedge, \sim, 0,1)$ such that satisfies $(\triangle 1),(\triangle 2),(\triangle 8)$ and $(\triangle 9) \triangle(x \rightarrow y) \leq \triangle x \rightarrow \triangle y$. 
Then, we have

(1) $\triangle(x \rightarrow y) \vee \triangle(y \rightarrow x)=1$,

(2) if $x \leq y$, then $\triangle x \leq \triangle y$,

(3) $(x \rightarrow y) \vee(y \rightarrow x)=1$.

(4) $\triangle(x \wedge y)=\triangle x \wedge \triangle y$,

(5) if $x \vee y$ and $\triangle x \vee \triangle y$ exist, then $\triangle(x \vee y)=\triangle x \vee \triangle y$,

(6) $\triangle(x \sim y) \leq \triangle x \sim \triangle y$.

PROOF:

(1) Suppose that $u$ is an upper bound of the set $\{\triangle(x \rightarrow y), \triangle(y \rightarrow x)\}$. By $(\triangle 8)$, Proposition 2.5 part (15) and part (6), we get $1=(x \rightarrow$ $y) \rightarrow u \leq((x \rightarrow y) \rightarrow u) \rightarrow u=1 \rightarrow u=u$. Hence $u=1$.

(2) It follows from Proposition 2.5 part $(4),(\triangle 9)$ and $(\triangle 1)$.

(3) Suppose that $u$ is an upper bound of the set $\{x \rightarrow y, y \rightarrow x\}$. Then $\triangle(x \rightarrow y) \leq \triangle u$ and $\triangle(y \rightarrow x) \leq \triangle u$ by part (2). By part (1), we obtain $\triangle u=1$. Hence $u=1$ by $(\triangle 2)$.

(4) By part (2), we have $\triangle(x \wedge y) \leq \triangle x \wedge \triangle y$. On the other hand, by Proposition 2.5 part $(17),(\triangle 9)$ and Proposition 2.5 part (16) $1=\triangle(x \rightarrow y) \vee \triangle(y \rightarrow x)=\triangle(x \rightarrow(x \wedge y)) \vee \triangle(y \rightarrow(x \wedge y)) \leq$ $(\triangle x \rightarrow \triangle(x \wedge y)) \vee(\triangle y \rightarrow \triangle(x \wedge y))=(\triangle x \wedge \triangle y) \rightarrow \triangle(x \wedge y)$. Thus $(\triangle x \wedge \triangle y) \leq \triangle(x \wedge y)$.

(5) By part (2), we have $\triangle x \vee \triangle y \leq \triangle(x \vee y)$. On the other hand, by part (4), $(\triangle 9)$, Proposition 2.5 part (15) and part (16), we obtain

$$
\begin{aligned}
\triangle(x \vee y) & =\triangle(((x \rightarrow y) \rightarrow y) \wedge((y \rightarrow x) \rightarrow x)) \\
& \leq((\triangle(x \rightarrow y) \rightarrow \triangle y) \wedge(\triangle(y \rightarrow x) \rightarrow \triangle x)) \\
& \leq((\triangle(x \rightarrow y) \rightarrow(\triangle x \vee \triangle y)) \wedge(\triangle(y \rightarrow x) \rightarrow(\triangle x \vee \triangle y)) \\
& \leq(\triangle(x \rightarrow y) \vee \triangle(y \rightarrow x)) \rightarrow(\triangle x \vee \triangle y)=(\triangle x \vee \triangle y) .
\end{aligned}
$$

(6) It follows from Proposition 2.5 part $(7),(\triangle 9)$, part (4) and part (2). 
COROLlary 4.9. Let $\triangle$ be a unary operation on bounded equality-algebra $\mathcal{A}=(A, \wedge, \sim, 0,1)$. Then $(A, \wedge, \sim, \triangle, 0,1)$ is a prelinear equality $\triangle$ - algebra if and only if it satisfies, for all $x, y, z \in A$.

$(\triangle 1) \triangle 1=1$

$(\triangle 2) \triangle x \leq x$,

$(\triangle 3) \triangle x \leq \triangle \triangle x$,

$(\triangle 8) \triangle(x \rightarrow y) \rightarrow z \leq(\triangle(y \rightarrow x) \rightarrow z) \rightarrow z$,

$(\triangle 9) \triangle(x \rightarrow y) \leq \triangle x \rightarrow \triangle y$,

( $\triangle 7) \triangle x \vee \neg \triangle x=1$, that is 1 is unique upper bound of the set $\{\triangle x, \neg \triangle x\}$ in $A$.

Proof: It follows from Proposition 4.5 and Proposition 4.8.

COROLlary 4.10. A prelinear equality $\triangle$-algebra is an equality $\triangle$-algebra satisfying the prelinearity. Moreover, it is a distributive lattice.

Proof: It follows from Proposition 4.8 part (3) an Theorem 2.4.

DeFinition 4.11. A $\triangle$-deductive system of an equality $\triangle$-algebra $(A, \wedge, \sim, \triangle, 0,1)$ is a deductive system $F$ of $(A, \wedge, \sim, 0,1)$ that satisfies for all $x \in F, \triangle x \in F$.

Example 4.12. Consider the prelinear equality $\triangle$-algebra $(A, \wedge, \sim, \triangle, 0,1)$ in Example 4.2 part (2). It is easy to see that $F_{1}=\{c, 1\}$ is a $\triangle$-deductive system of $A$. Also, $F_{2}=\{c, 1\}$ is a deductive system of $A$ but it is not a $\triangle$-deductive system.

Let $(A, \wedge, \sim, \triangle, 0,1)$ be an equality $\triangle$-algebra and $X$ be a nonempty subset of $A$. We denote by $\langle X\rangle_{\triangle}$ the $\triangle$-deductive system of $A$ generated by $X$, that is, $\langle X\rangle_{\triangle}$ is the smallest $\triangle$-deductive system of $A$ containing $X$. If $F$ is a $\triangle$-deductive system of $A$ and $x \notin F$, then $\langle F, x\rangle_{\triangle}:=\langle F \cup\{x\}\rangle_{\triangle}$.

THEOREM 4.13. Let $X$ be a nonempty subset of an equality $\triangle$-algebra $(A, \wedge, \sim, \triangle, 0,1)$. Then

(i) $\langle X\rangle_{\triangle}=\left\{x \in A \mid \exists n \in \mathbb{N}, y_{1}, \ldots, y_{n} \in A \ni \triangle y_{1} \rightarrow\left(\triangle y_{2} \rightarrow \ldots\left(\triangle y_{n} \rightarrow\right.\right.\right.$ $x) \ldots)=1\}$, 
(ii) If $F$ is a deductive system of $A$ and $S \subseteq A$, then $\langle F \cup S\rangle_{\triangle}=\{x \in$ $\left.A \mid \exists n \in \mathbb{N}, s_{1}, \ldots, s_{n} \in S \ni \triangle s_{1} \rightarrow\left(\triangle s_{2} \rightarrow \ldots\left(\triangle s_{n} \rightarrow x\right) \ldots\right) \in F\right\}$,

(iii) $\langle a\rangle_{\triangle}=\left\{x \in A \mid \exists n \in \mathbb{N}, \triangle x \rightarrow^{n} a=1\right\}$.

Proof: The proof is straightforward.

DEFinition 4.14. Let $\mathcal{A}_{\triangle}=(A, \wedge, \sim, \triangle, 0,1)$ be an equality $\triangle$-algebra and $\theta$ be a congruence on an equality-algebra $(A, \wedge, \sim, 0,1)$. Then $\theta$ is called a $\triangle$-congruence on $\mathcal{A}_{\triangle}$, if $(x, y) \in \theta$, then $(\triangle x, \triangle y) \in \theta$, for any $x, y \in A$.

Proposition 4.15. Let $\mathcal{A}_{\triangle}=(A, \wedge, \sim, \triangle, 0,1)$ be an equality $\triangle$-algebra and let $F$ be a $\triangle$-deductive system. Put $(x, y) \in \theta_{F}$ iff $x \sim y \in F$. Then

(i) $\theta_{F}$ is a $\triangle$-congruence and the corresponding quotient algebra $\left(\mathcal{A} / \theta_{\mathcal{F}}\right)_{\triangle}=\left(A / \theta_{F}, \wedge, \sim, \triangle, 1\right)$ is an equality $\triangle$-algebra, where for every $x, y \in A,[x] \wedge[y]:=[x \wedge y],[x] \sim[y]:=[x \sim y], \triangle[x]:=[\triangle x]$ and $1:=[1]$.

(ii) $\left(\mathcal{A} / \theta_{\mathcal{F}}\right)_{\triangle}$ is linearly ordered iff $F$ is a prime $\triangle$-deductive system of $\mathcal{A}$.

(iii) if $\mathcal{A}_{\triangle}$ is a prelinear equality $\triangle$-algebra, then $\left(\mathcal{A} / \theta_{\mathcal{F}}\right)_{\triangle}$ is a prelinear equality $\triangle$-algebra.

PROOF: The proof is straightforward.

Let $\mathcal{A}=(A, \wedge, \sim, 0,1)$ be an equality-algebra. For $x, y \in A$ and $n \in \mathbb{N}_{0}$, we define $x \rightarrow^{n} y$ inductively as follows:

$$
\begin{aligned}
& x \rightarrow^{0} y=y, \\
& x \rightarrow^{n} y=x \rightarrow\left(x \rightarrow^{n-1} y\right) \text { for } n \geq 1 .
\end{aligned}
$$

The proof of the following lemma is similar to the proof of lemma 3.3 in [15].

LEMmA 4.16. Let $\mathcal{A}_{\triangle}=(A, \wedge, \sim, \triangle, 0,1)$ be an equality $\triangle$-algebra satisfying prelinearity and $P$ be a prime $\triangle$-deductive system of $\mathcal{A}$. If $x \rightarrow^{n} z \in P$ and $y \rightarrow^{m} z \in P$ for $m, n \in \mathbb{N}$, then $(x \vee y) \rightarrow^{r} z \in P$ for some $r \in \mathbb{N}$.

Proof: Suppose that $l=\max \{n, m\}$. Then $x \rightarrow^{l} z, y \rightarrow^{l} z \in P$. We will prove by induction on $l$. For $l=1$, we have $(x \vee y) \rightarrow z=(x \rightarrow z) \wedge(y \rightarrow$ $z) \in P$ by Proposition 2.5 part (16). Thus $r=1$. 
Now, suppose that the statement holds for all $k \in N$ with $k \leq l$ and $x \rightarrow^{l+1} z, y \rightarrow^{l+1} z \in P$. Since

$$
\begin{aligned}
y \rightarrow^{l+1} z \leq x \rightarrow^{l}\left(y \rightarrow^{l+1} z\right) & =x \rightarrow^{l}\left(y \rightarrow^{l}\left(y \rightarrow^{l} z\right)\right) \\
& =y \rightarrow\left(x \rightarrow^{l}\left(y \rightarrow^{l+1} z\right)\right), \\
x \rightarrow^{l+1} z \leq x \rightarrow^{l+1}\left(y \rightarrow^{l} z\right) & =x \rightarrow\left(x \rightarrow^{l}\left(y \rightarrow^{l} z\right),\right.
\end{aligned}
$$

then $y \rightarrow\left(x \rightarrow^{l}\left(y \rightarrow^{l+1} z\right)\right), x \rightarrow\left(x \rightarrow^{l}\left(y \rightarrow^{l} z\right) \in P\right.$. Using Proposition 2.5 part (11) and part (16), we get

$$
\begin{aligned}
x \rightarrow(x & \left.\rightarrow^{l-1}\left(y \rightarrow^{l}((x \vee y) \rightarrow z)\right)\right)=(x \vee y) \rightarrow\left(\left(x \rightarrow^{l}\left(y \rightarrow^{l} z\right)\right.\right. \\
& =\left[y \rightarrow\left(x \rightarrow^{l}\left(y \rightarrow^{l+1} z\right)\right)\right] \wedge\left[x \rightarrow\left(x \rightarrow^{l}\left(y \rightarrow^{l} z\right)\right] \in P .\right.
\end{aligned}
$$

By Proposition 2.5 part (15)

$$
\begin{aligned}
y \rightarrow^{l+1} z & \leq y \rightarrow^{l+1}((x \vee y) \rightarrow z) \\
& \leq x \rightarrow^{l-1}\left(y \rightarrow^{l+1}((x \vee y) \rightarrow z)\right) \\
& =y \rightarrow\left(x \rightarrow^{l-1}\left(y \rightarrow^{l}((x \vee y) \rightarrow z)\right) .\right.
\end{aligned}
$$

Thus

$$
y \rightarrow\left(x \rightarrow^{l-1}\left(y \rightarrow^{l}((x \vee y) \rightarrow z)\right) \in P .\right.
$$

By Proposition 2.5 part (11) and part (16), (2.1) and (2.2), we get $x \rightarrow\left(x \rightarrow^{l-2}\left(y \rightarrow^{l}\left((x \vee y) \rightarrow^{2} z\right)\right)\right)=(x \vee y) \rightarrow\left(\left(x \rightarrow^{l-1}\left(y \rightarrow^{l}(x \vee y) \rightarrow\right.\right.\right.$ $z))=\left[y \rightarrow\left(x \rightarrow^{l-1}\left(y \rightarrow^{l}((x \vee y) \rightarrow z)\right)\right) \wedge x\right] \rightarrow\left[\left(x \rightarrow^{l-1}\left(y \rightarrow^{l-1}\right.\right.\right.$ $((x \vee y) \rightarrow z))] \in P$. By repeating this, we get

$$
\left.y \rightarrow^{l}\left((x \vee y) \rightarrow^{l+1} z\right)\right) \in P .
$$

by interchanging $x, y$, we obtain

$$
\left.x \rightarrow^{l}\left((x \vee y) \rightarrow^{l+1} z\right)\right) \in P .
$$

Using induction hypothesi to (2.3) and (2.4), there exists $s \in N$ such that $(x \vee y) \rightarrow^{s+l+1} z=(x \vee y) \rightarrow^{s}\left((x \vee y) \rightarrow^{l+1} z\right) \in P$. Hence $r=s+l+1$.

Proposition 4.17. Let $(A, \wedge, \sim, \triangle, 0,1)$ be a prelinear equality $\triangle$-algebra and let $a \in A, a \neq 1$. Then there is a prime $\triangle$-deductive system $F$ on $A$ not containing $a$. 
Proof: Suppose that $\mathcal{P}=\{F: F$ is a proper $\triangle$-deductive system and $a \notin F\}$. Then $\mathcal{P}$ is a partially set under inclusion relation. Since $\{1\} \in \mathcal{P}$, then $\mathcal{P}$ is a nonempty set. It is easy to see that every chain in $\mathcal{P}$ has an upper bound in $\mathcal{P}$. By Zorn's Lemma, there exists a maximal element $P$ in $\mathcal{P}$. Since $P \in \mathcal{P}$, then $P$ is a $\triangle$-deductive system of $A$ not containing $a$. We will prove that $P$ is prime. If $P$ is not prime, then there exist $x, y \in A$ such that $x \rightarrow y, y \rightarrow x \notin P$. Since $P$ is strictly contained in $\langle P, x \rightarrow y\rangle_{\triangle}$ and $\langle P, y \rightarrow x\rangle_{\triangle}$, then $\langle P, x \rightarrow y\rangle_{\triangle} \notin \mathcal{P}$ and $\langle P, y \rightarrow x\rangle_{\triangle} \notin \mathcal{P}$ by the maximality of $P$. Thus $a \in\langle P, y \rightarrow x\rangle_{\triangle}$ and $a \in\langle P, x \rightarrow y\rangle_{\triangle}$. Then there exist $n, m \in N$ such that $\triangle(x \rightarrow y) \rightarrow^{n} a \in P$ and $\triangle(y \rightarrow x) \rightarrow^{m} a \in P$ by Theorem 4.13 part (iii). By Lemma 4.16, there exists $r \in \mathbb{N}$ such that $(\triangle(x \rightarrow y) \vee \triangle(y \rightarrow x)) \rightarrow^{r} a \in P$. By Proposition 4.8 part (1), we obtain $a \in P$ which is a contradiction.

Proposition 4.18. Each prelinear equality $\triangle$-algebra is a subalgebra of the direct product of a system of linearly ordered equality $\triangle$-algebra.

Proof: Suppose that $\mathcal{P}$ is the class of all prime $\triangle$-deductive systems of a prelinear equality $\triangle$-algebra $(A, \wedge, \sim, \triangle, 0,1)$. Then $B=\prod_{\theta \in \mathcal{P}} A / \theta_{F}$ is a direct product of linearly ordered equality $\triangle^{-}$algebra by Proposition 4.15 part (iii). Define $f: A \rightarrow B$ by $f(x)=\left\{x / \theta_{F}: F \in \mathcal{P}\right\}$. It is easy to prove that $f$ preserves operations. We will prove that $f$ is one to one. Suppose that $x, y \in A$ such that $x \neq y$. Then $x \not \leq y$ or $y \not \leq x$. Suppose that $x \not \leq y$. Then $x \rightarrow y \neq 1$. By Proposition 4.17, there exists a prime $\triangle$-deductive system $F$ such that $x \rightarrow y \notin F$. Thus $x / \theta_{F} \not \leq y / \theta_{F}$ in $A / \theta_{F}$. So $x / \theta_{F} \neq y / \theta_{F}$ in $A / \theta_{F}$. Hence $f(x) \neq f(y)$.

\section{Prelinear equality $\triangle \operatorname{logic}$}

In this section, we introduce the logic corresponding to prelinear equality $\triangle^{-}$ algebras and prove that the resulting logic, i.e. propositional prelinear equality $\triangle \operatorname{logic} \mathcal{E} \mathcal{L}_{\triangle}$ is sound and complete with respect to the variety of prelinear equality $\triangle$-algebras.

DEFINITION 5.1.

(i) The language of propositional prelinear equality $\triangle \operatorname{logic} \mathcal{E} \mathcal{L}_{\triangle}$ is the language of propositional equality logic $\mathcal{E} \mathcal{L}$ expanded by the unary connective $\Delta$ and the truth constant $\perp$. 
(ii) Formulas of $\mathcal{E} \mathcal{L}_{\triangle}$ are defined in the following way:

each formula of $\mathcal{E} \mathcal{L}$ is a formula of $\mathcal{E} \mathcal{L}_{\triangle}, \perp$ is a formula and if $\varphi$ is a formula, then $\Delta(\varphi)$ is a formula. Disjunction and negation connectives are defined as the following short:

$$
\varphi \sqcup \psi:=((\varphi \Rightarrow \psi) \Rightarrow \psi) \sqcap((\psi \Rightarrow \varphi) \Rightarrow \varphi), \quad \neg \varphi=: \varphi \Rightarrow \perp .
$$

The set of all formulas of $\mathcal{E} \mathcal{L}_{\triangle}$ is denoted by $\mathcal{F}_{\triangle}$.

(iii) The logical axioms of $\mathcal{E} \mathcal{L}_{\triangle}$ consist of the logical axioms of $\mathcal{E} \mathcal{L}$ plus the following axioms :

(E10) $(\varphi \sqcap \perp) \equiv \perp$,

$(\mathrm{E} \Delta 1) \Delta \top$

$(\mathrm{E} \Delta 2) \Delta \varphi \Rightarrow \varphi$

$(\mathrm{E} \Delta 3) \Delta \varphi \Rightarrow \Delta \Delta \varphi$

$(\mathrm{E} \Delta 4)(\Delta(\varphi \Rightarrow \psi) \Rightarrow \chi) \Rightarrow((\Delta(\psi \Rightarrow \varphi) \Rightarrow \chi) \Rightarrow \chi)$,

$(\mathrm{E} \Delta 5) \Delta(\varphi \Rightarrow \psi) \Rightarrow(\Delta \varphi \Rightarrow \Delta \psi)$,

$(\mathrm{E} \Delta 6)(\Delta \varphi \Rightarrow \neg \Delta \varphi) \Rightarrow \neg \Delta \varphi$,

$(\mathrm{E} \Delta 7)(\neg \Delta \varphi \Rightarrow \Delta \varphi) \Rightarrow \Delta \varphi$.

(iv) The inference rules of $\mathcal{E} \mathcal{L}_{\triangle}$ are (EA), (MP) and generalization (Gen): from $\varphi$ derive $\Delta \varphi$.

Definition 5.2. Let $\mathcal{A}_{\triangle}=(A, \wedge, \sim, \triangle, 0,1)$ be a prelinear equality $\triangle^{-}$ algebra. An $\mathcal{A}_{\triangle}$-evaluation of formulas is a mapping $e: \mathcal{F}_{\triangle} \rightarrow A$, defined as follows:

(1) $e(\perp)=0$,

(2) $e(\top)=1$,

(3) $e(\Delta \varphi)=\Delta e(\varphi)$,

(4) $e(\varphi \sqcap \psi)=e(\varphi) \wedge e(\psi)$,

(5) $e(\varphi \equiv \psi)=e(\varphi) \sim e(\psi)$,

for all formulas $\varphi, \psi \in \mathcal{F}_{\triangle}$. A formula $\varphi$ is a $\mathcal{A}_{\triangle}$-tautology if $e(\varphi)=1$ for

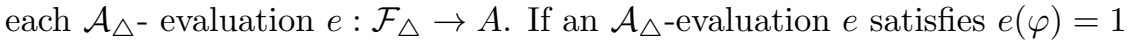
for every $\varphi$ in theory $\Gamma$, then it is called an $\mathcal{A}_{\triangle}$-model of $\Gamma$.

The propositional prelinear equality $\triangle \operatorname{logic} \mathcal{E} \mathcal{L}_{\triangle}$ is an extension of the propositional equality $\operatorname{logic} \mathcal{E} \mathcal{L}$. Thus every the theorems and inferences of 
$\mathcal{E} \mathcal{L}$ is valid in $\mathcal{E} \mathcal{L}_{\triangle}$. In the following Lemma, we prove properties that we will use in the strong completeness of $\mathcal{E} \mathcal{L}_{\triangle}$.

Lemma 5.3. Let $\varphi, \psi, \chi \in \mathcal{F}$ be formulas. $\mathcal{E} \mathcal{L}_{\triangle}$ proves the following properties:

(1) $\{\Delta(\varphi \Rightarrow \psi) \Rightarrow \chi, \Delta(\psi \Rightarrow \varphi) \Rightarrow \chi\} \vdash \chi$,

(2) $\{(\varphi \Rightarrow \psi) \Rightarrow \chi,(\psi \Rightarrow \varphi) \Rightarrow \chi\} \vdash \chi$,

$(3) \vdash((\varphi \Rightarrow \psi) \sqcap(\psi \Rightarrow \varphi)) \Rightarrow(\varphi \equiv \psi)$,

(4) $\vdash \varphi \equiv \psi \vdash \Delta \varphi \equiv \Delta \psi$,

(5) $\vdash \Delta \top \equiv \top$.

Proof:

(i) The results follows from assumptions, (E $\Delta 4)$ and (MP).

(ii) Suppose that $\Gamma=\{(\varphi \Rightarrow \psi) \Rightarrow \chi,(\psi \Rightarrow \varphi) \Rightarrow \chi\}$. By assumption, (Gen), (E $\Delta 5)$ and (MP), we have $\Gamma \vdash \Delta(\varphi \Rightarrow \psi) \Rightarrow \Delta \chi$ and $\Gamma \vdash$ $\Delta(\psi \Rightarrow \varphi) \Rightarrow \Delta \chi$. Using part (1), we obtain $\Gamma \vdash \chi$.

(iii) Using Proposition 3.13 part (9), Proposition 3.8 part (8) and part (7), we get $\vdash(\varphi \Rightarrow \psi) \Rightarrow[((\varphi \Rightarrow \psi) \sqcap(\psi \Rightarrow \varphi)) \Rightarrow(\varphi \equiv \psi)]$, $\vdash(\psi \Rightarrow \varphi) \Rightarrow[((\varphi \Rightarrow \psi) \sqcap(\psi \Rightarrow \varphi)) \Rightarrow(\varphi \equiv \psi)]$. Applying part $(2)$, the result is obtained.

(iv) It is easy to prove by assumption, Proposition 3.13 part (11), (Gen), (E $\Delta 5)$, (MP), Proposition 3.7 part (7) and then part (3) and (MP).

(v) Using (EL5), we have $\vdash(\Delta T \equiv \top) \equiv \Delta T$. By (EL6) and (EA), we obtain $\vdash \Delta T \equiv(\Delta T \equiv T)$. Applying $(\mathrm{E} \Delta 1)$ and $(\mathrm{EA})$, we have $\vdash \Delta \top \equiv \top$.

Proposition 5.4. Let $\Gamma$ be a theory over the propositional equality logic $\mathcal{E} \mathcal{L}_{\triangle}$. Then algebra $\mathcal{M}_{\Gamma}=\left(M_{\Gamma}, \wedge, \sim, \triangle, 0,1\right)$ is a prelinear equality $\triangle^{-}$ algebra where $1=[\top]_{\Gamma}, 0=[\perp]_{\Gamma}, \triangle[\psi]_{\Gamma}:=[\Delta(\varphi)]_{\Gamma},[\varphi]_{\Gamma} \wedge[\psi]_{\Gamma}:=[\varphi \sqcap \psi]_{\Gamma}$ and $[\varphi]_{\Gamma} \sim[\psi]_{\Gamma}:=[\varphi \equiv \psi]_{\Gamma}$.

Proof: Let $\Gamma$ be a theory over the propositional equality logic $\mathcal{E} \mathcal{L}_{\triangle}$. Since $\Gamma$ be a theory over the propositional equality logic $\mathcal{E} \mathcal{L}$, then $\left(M_{\Gamma}, \wedge, \sim, 1\right)$ is an equality algebra by Proposition 3.11. By (E10), 
$\left(M_{\Gamma}, \wedge, \sim, 0,1\right)$ is bounded. By Lemma $5.3, \triangle$ is well defined. Using Lemma 5.3 and Corollary 4.9 , it is easy to prove $\mathcal{M}_{\Gamma}=\left(M_{\Gamma}, \wedge, \sim, \triangle, 0,1\right)$ is a prelinear equality $\triangle$ - algebra.

DeFinition 5.5. Let $\Gamma$ be a theory over the propositional equality logic $\mathcal{E} \mathcal{L}_{\triangle}$.

(1) A theory $\Gamma$ is contradictory if for some $\varphi, \Gamma$ proves $\varphi$ and $\Gamma$ proves $\neg \varphi$. $\Gamma$ is consistent if it is not contradictory.

(2) $\Gamma$ is complete if for every pair $\varphi$ and $\psi$ of formulas, then $\Gamma \vdash \varphi \Rightarrow \psi$ or $\Gamma \vdash \psi \Rightarrow \varphi$.

Lemma 5.6. Let $\Gamma$ be a theory over the propositional equality logic $\mathcal{E} \mathcal{L}_{\triangle}$.

(1) $\Gamma$ is complete iff the prelinear equality $\triangle$-algebra $\mathcal{M}_{\Gamma}$ is linearly ordered.

(2) If $\Gamma \nvdash \varphi$, then there exists a consistent complete supertheory $T \subseteq T^{\prime}$ such that $T^{\prime} \forall \varphi$.

ProOF:

(1) It is obvious.

(2) It follows similarly with the proof of Proposition 4.17.

Theorem 5.7 (Strong completeness). Let $\Gamma$ be a theory over $\mathcal{E} \mathcal{L}_{\triangle}$ and $\varphi$ be a formula. Then the following are equivalent:

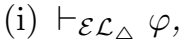

(ii) For each linearly ordered equality $\triangle$-algebra $\mathcal{A}$ and each $\mathcal{A}$-model e of $\Gamma, e(\varphi)=1$,

(iii) For each prelinear equality $\triangle$-algebra $\mathcal{A}$ and each $\mathcal{A}$-model $e$ of $\Gamma, e(\varphi)=1$.

PROOF:

(i) $\Rightarrow$ (ii) This is because all axioms of $\mathcal{E} \mathcal{L}_{\triangle}$ are true in all $\mathcal{A}$-models of $\Gamma$, axioms of $\Gamma$ are true in all models of $\Gamma$ by the definition of a model and the inference rules of $\mathcal{E} \mathcal{L}_{\triangle}$ are sound in the following sense:

(1) If for all prelinear equality $\triangle$-algebra $\mathcal{A}$ and for all $\mathcal{A}$-model $e$ of $\Gamma$, $e(\varphi)=1$ and $e(\varphi \equiv \psi)=1$, then for all prelinear equality $\triangle$-algebra $\mathcal{A}$ and for all $\mathcal{A}$-model $e$ of $\Gamma, e(\psi)=1$. 
(2) If for all prelinear equality $\triangle$-algebra $\mathcal{A}$ and for all $\mathcal{A}$-model $e$ of $\Gamma$, $e(\varphi)=1$ and $e(\varphi \Rightarrow \psi)=1$, then for all prelinear equality $\triangle$-algebra $\mathcal{A}$ and for all $\mathcal{A}$-model $e$ of $\Gamma, e(\psi)=1$.

(3) If for all prelinear equality $\triangle$-algebra $\mathcal{A}$ and for all $\mathcal{A}$-model $e$ of $\Gamma, e(\varphi)=1$, then for all prelinear equality $\triangle$-algebra $\mathcal{A}$ and for all $\mathcal{A}$-model $e$ of $\Gamma, e(\Delta(\varphi))=1$.

(ii) $\Rightarrow$ (i) Suppose that $\Gamma \nvdash \varphi$. Then then there exists a consistent complete supertheory $\Gamma \subseteq \Gamma^{\prime}$ such that $\Gamma^{\prime} \nvdash \varphi$ by Lemma 5.6 part (2). Since $\Gamma^{\prime}$ is complete, then the prelinear equality $\triangle^{-}$algebra $\mathcal{M}_{\Gamma}$ is linearly ordered. For each propositional variable $p$, define $e(\psi)=[\psi]_{\Gamma^{\prime}}$. Then we have an $\mathcal{M}_{\Gamma}$-model of $\Gamma$ such that $e(\varphi)<1$, which is a contradiction.

(ii) $\Rightarrow$ (iii) follows from Proposition 4.17.

(iii) $\Rightarrow$ (ii) is obvious.

Acknowledgements The author would like to thank the referees for a number of helpful comments and suggestions.

\section{References}

[1] W. J. Blok, D. Pigozzi, Algebraizable logics, vol. 77, American Mathematical Society (1989), DOI: http://dx.doi.org/10.1090/memo/0396.

[2] W. J. Blok, D. Pigozzi, Abstract algebraic logic and the deduction theorem (2001), URL: https://orion.math.iastate.edu/dpigozzi/papers/ aaldedth.pdf.

[3] R. Borzooei, F. Zebardast, M. Aaly Kologani, Some types of filters in equality algebras, Categories and General Algebraic Structures with Applications, vol. 7 (Special Issue on the Occasion of Banaschewski's 90th Birthday (II)) (2017), pp. 33-55, DOI: http://dx.doi.org/10.1007/s00500005-0534-4.

[4] R. A. Borzooei, M. Zarean, O. Zahiri, Involutive equality algebras, Soft Computing, vol. 22(22) (2018), pp. 7505-7517, DOI: http://dx.doi.org/10. 1007/s00500-018-3032-1.

[5] J. R. Büchi, T. M. Owens, Skolem rings and their varieties, [in:] The Collected Works of J. Richard Büchi, Springer (1990), pp. 161-221, DOI: http://dx.doi.org/10.1007/978-1-4613-8928-6-11. 
[6] L. C. Ciungu, Internal states on equality algebras, Soft computing, vol. 19(4) (2015), pp. 939-953, DOI: http://dx.doi.org/10.1007/s00500-0141494-3.

[7] J. Czelakowski, Protoalgebraic logics, [in:] Protoalgebraic Logics, Springer (2001), pp. 69-122, DOI: http://dx.doi.org/10.1007/978-94-0172807-2-3.

[8] M. Dyba, M. El-Zekey, V. Novák, Non-commutative first-order EQ-logics, Fuzzy Sets and Systems, vol. 292 (2016), pp. 215-241, DOI: http://dx. doi.org/10.1016/j.fss.2014.11.019.

[9] M. Dyba, V. Novák, EQ-logics: Non-commutative fuzzy logics based on fuzzy equality, Fuzzy Sets and Systems, vol. 172(1) (2011), pp. 13-32, DOI: http://dx.doi.org/10.1016/j.fss.2010.11.011.

[10] M. El-Zekey, Representable good EQ-algebras, Soft Computing, vol. 14(9) (2010), pp. 1011-1023, DOI: http://dx.doi.org/10.1007/s00500-009-0491-4.

[11] M. El-Zekey, V. Novák, R. Mesiar, On good EQ-algebras, Fuzzy Sets and Systems, vol. 178(1) (2011), pp. 1-23, DOI: http://dx.doi.org/10.1016/j. fss.2011.05.011.

[12] S. Ghorbani, Monadic pseudo-equality algebras, Soft Computing, vol. 23(24) (2019), pp. 12937-12950, DOI: http://dx.doi.org/10.1007/ s00500-019-04243-5.

[13] S. Jenei, Equality algebras, Studia Logica, vol. 100(6) (2012), pp. 12011209, DOI: http://dx.doi.org/10.1007/s11225-012-9457-0.

[14] S. Jenei, L. Kóródi, On the variety of equality algebras, [in:] Proceedings of the 7th conference of the European Society for Fuzzy Logic and Technology, Atlantis Press (2011), pp. 153-155, DOI: http://dx.doi.org/ 10.2991/eusflat.2011.1.

[15] J. Kühr, Pseudo BCK-semilattices, Demonstratio Mathematica, vol. 40(3) (2007), pp. 495-516, DOI: http://dx.doi.org/10.1515/dema-20070302.

[16] V. Novák, On fuzzy type theory, Fuzzy Sets and Systems, vol. 149(2) (2005), pp. 235-273, DOI: http://dx.doi.org/10.1016/j.fss.2004.03.027.

[17] V. Novák, EQ-algebras: primary concepts and properties, [in:] Proceedings of International Joint Czech Republic-Japan \& Taiwan-Japan Symposium, Kitakyushu, Japan, August 2006 (2006), pp. 219-223. 
[18] V. Novák, EQ-algebra-based fuzzy type theory and its extensions, Logic Journal of the IGPL, vol. 19(3) (2011), pp. 512-542, DOI: http: //dx.doi.org/10.1093/jigpal/jzp087.

[19] V. Novák, B. De Baets, EQ-algebras, Fuzzy Sets and Systems, vol. 160(20) (2009), pp. 2956-2978, DOI: http://dx.doi.org/10.1016/j.fss.2009. 04.010 .

[20] R. Suszko, Non-Fregean logic and theories, Analele Universitatii Bucuresti, Acta Logica, vol. 11 (1968), pp. 105-125.

[21] J. T. Wang, X. L. Xin, Y. B. Jun, Very true operators on equality algebras, Journal of Computational Analysis and Applications, vol. 24(3) (2018), DOI: http://dx.doi.org/10.1515/math-2016-0086.

[22] M. Zarean, R. A. Borzooei, O. Zahiri, On state equality algebras, Quasigroups and Related Systems, vol. 25(2) (2017), pp. 307-326.

[23] F. Zebardast, R. A. Borzooei, M. A. Kologani, Results on equality algebras, Information Sciences, vol. 381 (2017), pp. 270-282, DOI: http://dx.doi. org/10.1016/j.ins.2016.11.027.

\section{Shokoofeh Ghorbani}

Shahid Bahonar University of Kerman

Faculty of Mathematics and Computer

Department of Pure Mathematics

Kerman, Iran

e-mail: sh.ghorbani@uk.ac.ir 